\title{
Computing Bounds for General Randic Coindex of Sum Graphs
}

\author{
Muhammad Javaid (1), ${ }^{1}$ Muhammad Ibraheem, ${ }^{1}$ and Ebenezer Bonyah $\mathbb{D}^{2}$ \\ ${ }^{1}$ Department of Mathematics, School of Science, University of Management and Technology, Lahore 54770, Pakistan \\ ${ }^{2}$ Department of Mathematics Education, Akenten Appiah-Menka University of Skills Training and Entrepreneurial Development, \\ Kumasi 00233, Ghana \\ Correspondence should be addressed to Ebenezer Bonyah; ebbonya@gmail.com
}

Received 15 November 2021; Accepted 6 December 2021; Published 26 December 2021

Academic Editor: Gohar Ali

Copyright (c) 2021 Muhammad Javaid et al. This is an open access article distributed under the Creative Commons Attribution License, which permits unrestricted use, distribution, and reproduction in any medium, provided the original work is properly cited.

\begin{abstract}
The physical and structural properties of molecular structure or graph such as boiling point, melting point, surface tension, or solubility are studied using topological index (TI). Topological index is a mathematical formula that can be applied to any graph which models some molecular structures. The various operations play an important role in graph theory such as joining, union, intersection, products, and subdivision. In this paper, we computed the bounds for general Randic coindex of $F$-sum graphs such as ( $S$-sum, $R$-sum, $Q$-sum, and $T$-sum) in the form of their factor graphs. At the end, results are illustrated by numerical table for the particular F-sum graphs.
\end{abstract}

\section{Introduction}

Graph theory is playing an important role in various sciences particularly in computer science and mathematical chemistry. The branch of mathematics which combines chemistry and graph theory is called chemical graph theory. The molecular structure descriptors have been used for quantifying information on molecules. This relates to characterizing physico-chemical, toxicologic, pharmacologic, biological, and other properties of chemical compounds by utilizing topological index (TI). TI is a mathematical formula that can be applied to any graph which models some molecular structures. It is an efficient mathematical method in avoiding laboratory experiments and time consumption $[1,2]$.

Actually, various TIs are introduced in order to describe physical and chemical properties of molecules. These indices are divided into different classes, namely, degree-based, distance-based, and polynomial-based, but the degree-based class is studied more than others, see the latest survey [3]. In 1947, Wiener calculated the boiling point of paraffin using a degree-based TI, see [4]. Gutman and Trinajstic calculated total $\pi$-electron energy of hydrocarbons using degree-based first and second Zagreb indices [5]. Li and Zheng provided the idea of first general Zagreb index (FGZI) [6].
The Randic index was proposed by Randic in 1975 and has been widely studied in different areas. Li and Shi [7] calculated the extremal values of Randic index and its higher-order, zeroth-order, and general form for the extremal graphs. Delorme et al. [8] proved a best-possible lower bound for triangle-free graph with minimum degree of graph and Gutman et al. [9] point out a hitherto unnoticed feature of a molecular graph for Randic index. Arizmendi and Arizmendi proved that graph energy is twice of the Randic index and investigated that the equality holds iff graph is the union of complete bipartite graphs [10]. Li and Yang calculated the bounds for graphs whose general Randic indices reach the maximum and minimum [11]. Furtula and Gutman calculated Randic energy value of the connected graph with a fixed number of vertices [12]. Gao and Lu calculated the sharp bounds for the unicyclic graphs [13] and Li et al. [14] computed the bounds for of chemical $(n-m)$ graphs for general Randic index.

$\mathrm{Ma}$ et al. [15] gave a brief review for the Randic from 1975 to date such as zeroth-order Randic indices, sumconnectivity indices, geometric-arithmetic indices, Randic spectrum and energy, harmonic index, Randic matrix, D-L-S generalization, Balaban index, and atom-bond connectivity 
index. Milovanovic et al. [16] wrote a note and calculated some mathematical properties of the general zeroth-order Randic coindex of graphs in [17].

In the development of new graphs, the various operations play an important role in graph theory such as joining, union, intersection, products, and subdivision. Yan et al. [18] listed five different operations on a graph $G$ such as line graph $L(G)$, subdivided graph $S(G)$, line superposition graph $Q(G)$, triangle parallel graph $R(G)$, and total graph $T(G)$, respectively; further, they computed Wiener index of these graphs. Eliasi and Taeri introduced the $F$-sum graphs such as $G_{1+F} G_{2}$, where $F \in\{S, R, Q, T\}$, and calculated the Wiener indices of graphs in [19]. Later on, many researchers worked on these $F$-sum graphs such as Sarala et al. computed first and second Zagreb indices [20], Imran et al. [21] investigated the bounds of degreebased topological indices such as bounds of Zagreb indices, multiple Zagreb indices, the atom-bond connectivity $(\mathrm{ABC})$ index, the forgotten topological index, the geometric-arithmetic (GA) index, and the Narumi-Katayama index, and $\mathrm{Li}$ et al. [22] computed bounds on general Randic indexes. Javaid et al. [23] calculated bounds for second Zagreb coindex, Akhter and Imran [24] calculated the forgotten topological index, Liu et al. [25] computing first general Zagreb index of operations on graphs, and Javaid et al. [26] calculated the Zagreb coindex and connection index of these graphs.

In this article, we investigated the sharp bounds for general Randic coindex of graphs that are obtained by using subdivisions related operations such as $\bar{R}_{\alpha}\left(G_{1+S} G_{2}\right)$, $\bar{R}_{\alpha}\left(G_{1+R} G_{2}\right), \bar{R}_{\alpha}\left(G_{1+Q} G_{2}\right)$, and $\bar{R}_{\alpha}\left(G_{1+T} G_{2}\right)$. The rest of the paper is organized as follows: Section 2 contains preliminaries and notations, Section 3 contains the main theorems of the work, and Section 4 contains conclusion of the work; further, the results are illustrated using examples for some particular F-sum graphs.

\section{Preliminaries}

Let $V(G)$ be nonempty set vertices and $E(G) \subseteq V(G) \otimes V(G)$ be the set of edges, then by combining both $V(G)$ and $E(G)$, a graph is formed that is denoted by $G=(V(G), E(G))$. The cardinality of vertex set is called order of graph and cardinality of edge set is known as size of the graph which are denoted by $|V(G)|=n$ and $|E(G)|=m$, respectively. Let $v \in V(G)$, then its degree is denoted by $d(v)$ and defined as number of edges incident on it. Let $G$ be a graph; its maximum and minimum degrees are denoted by $\Delta(G)$ and $\delta(G)$, respectively. For any graph $G$, its complement is denoted by $\bar{G}$ and defined as $u v \in E(\bar{G})$ iff $u v \notin E(\bar{G})$. Gutman and Trinajstic [5] introduced degree-based TIs known as Zagreb indices. Now, we define first and second Zagreb indices and coindices for any $G$,

$$
\begin{aligned}
& M_{1}(G)=\sum_{x_{1} x_{2} \in E(G)}\left[d_{G}\left(x_{1}\right)+d_{G}\left(x_{2}\right)\right], \\
& M_{2}(G)=\sum_{x_{1} x_{2} \in E(G)}\left[d_{G}\left(x_{1}\right) d_{G}\left(x_{2}\right)\right], \\
& \bar{M}_{1}(G)=\sum_{x_{1} x_{2} \notin E(G)}\left[d_{G}\left(x_{1}\right)+d_{G}\left(x_{2}\right)\right], \\
& \bar{M}_{2}(G)=\sum_{x_{1} x_{2} \notin E(G)}\left[d_{G}\left(x_{1}\right) d_{G}\left(x_{2}\right)\right] .
\end{aligned}
$$

Li and Zheng [6] introduced the first general Zagreb index that is defined as

$$
M_{1}^{\alpha}(G)=\sum_{x_{1} x_{2} \in E(G)}\left[d_{G}\left(x_{1}\right)^{\alpha-1}+d_{G}\left(x_{2}\right)^{\alpha-1}\right] .
$$

By putting $\alpha=2$ and $\alpha=3$, we obtained the first Zagreb index and forgotten index, respectively.

Zhou and Trinajstic [27] introduced the general sumconnectivity index (GSCI) denoted by $\chi_{k}(G)$ after that general sum-connectivity coindex was introduced which is denoted by $\bar{\chi}_{k}(G)$; these are defined as

$$
\begin{aligned}
& \chi_{k}(G)=\sum_{x_{1} x_{2} \in E(G)}\left[d_{G}\left(x_{1}\right)+d_{G}\left(x_{2}\right)\right]^{k}, \\
& \bar{\chi}_{k}(G)=\sum_{x_{1} x_{2} \notin E(G)}\left[d_{G}\left(x_{1}\right)+d_{G}\left(x_{2}\right)\right]^{k} .
\end{aligned}
$$

Bollobas and Erdos [28] introduced the concept of general Randic coindex denoted $R_{\alpha}(G)$; its coindex is denoted by $\bar{R}_{\alpha}(G)$ which is defined as

$$
\begin{aligned}
& R_{\alpha}(G)=\sum_{x_{1} x_{2} \in E(H)}\left[d_{G}\left(x_{1}\right) d_{G}\left(x_{2}\right)\right]^{\alpha}, \\
& \bar{R}_{\alpha}(G)=\sum_{x_{1} x_{2} \notin E(G)}\left[d_{G}\left(x_{1}\right) d_{G}\left(x_{2}\right)\right]^{\alpha} .
\end{aligned}
$$

The binomial and trinomial theorems are very important while expanding expression of those described as

$$
\begin{aligned}
\left(y_{1}+y_{2}\right)^{n} & =\sum_{i=0}^{n}\left(\begin{array}{c}
n \\
i
\end{array}\right) y_{1}^{n-i} y_{2}^{i}, \\
\left(y_{1}+y_{2}+y_{3}\right)^{n} & =\sum_{\substack{m, k, l \\
m, k, m+k+l=n}} P_{m, k, l} y_{1}^{m} y_{2}^{k} y_{3}^{l},
\end{aligned}
$$

where $P_{m, k, l}=(m+k+l) / m ! k ! l !$.

Let $G$ be a graph, then $S(G)$ is known as edge subdivision graph that is obtained inserting a vertex in each edge of $G$, $R(G)$ is called triangle parallel graph that is obtained from $S(G)$ by joining an edge between the adjacent vertices of $G$, $Q(G)$ is called superposition graph obtained from $S(G)$ by joining an edge between the pairs of new vertices which are 
on the adjacent edges of $G$, and $T(G)$ is called total graph obtained by performing both operations of $R(G)$ and $Q(G)$ on $S(G)$.

Let $G_{1}$ and $G_{2}$ be two simple connected graphs, then their $F$-sum graph with vertex set $V\left(G_{1+F} G_{2}\right)=$ $V\left(G_{1}\right) \cup E\left(G_{1}\right) \times V\left(G_{2}\right)$ and $\left(s_{1}, s_{2}\right)\left(x_{1}, x_{2}\right) \in E\left(G_{1+F} G_{2}\right)$ iff $s_{1}=x_{1} \in V\left(G_{1}\right)$ and $s_{2} \backsim x_{2} \in G_{2}, \quad s_{2}=x_{2} \in V\left(G_{2}\right)$ and $s_{1} \backsim x_{1} \in F\left(G_{1}\right)$, where $F \in\{S, R, Q, T\}$, For details, see Figures 1 and 2 .

\section{Main Results}

This section contains results of the bounds for general Randic coindex.

$$
\begin{aligned}
& \alpha_{1}=\sum_{\substack{s_{1} s_{2} \notin E\left(S\left(G_{1}\right)\right) \\
s_{1} \in V\left(G_{1}\right) \\
s_{2} \in V\left(S\left(G_{1}\right)-V\left(G_{1}\right)\right)}}\left(d_{S\left(G_{1}\right)}\left(s_{1}\right)\right)\left(d_{S}\left(s_{2}\right)\right), \\
& \alpha_{2}=\sum_{s_{1}, s_{2} \in V\left(S\left(G_{1}\right)-V\left(G_{1}\right)\right)} \sum_{x_{1}, x_{2} \in V_{G_{2}}}\left[d\left(s_{1}, x_{1}\right)\left(s_{2}, x_{2}\right)\right]^{\alpha} .
\end{aligned}
$$

Theorem 1. Let $G_{1+S} G_{2}$ be an S-sum graph, then its general Randic coindex $\bar{R}_{\alpha}\left(G_{1+S} G_{2}\right)$ is given as

$$
\mathrm{LB} \leq \bar{R}_{\alpha}\left(G_{1+S} G_{2}\right) \leq \mathrm{UB},
$$

where

$$
\begin{aligned}
\mathrm{LB}= & \sum_{\substack{m, k, l \\
m, k, m+k+l=\alpha}} P_{m, k, l}\left[M_{1}^{2 m+k}\left(G_{1}\right) \bar{\chi}_{k}\left(G_{2}\right) \delta\left(G_{2}\right)\right]^{2 l}+\delta\left(G_{1}\right)^{2 m}\left[M_{1}^{2 l+k}\left(G_{2}\right)\left(\chi_{k}\left(G_{1}\right)+\bar{\chi}_{k}\left(G_{1}\right)\right)\right]+\delta\left(G_{2}\right)^{2 l} \\
& \left(M_{1}^{k}\left(G_{1}\right)+\bar{M}_{1}^{k}\left(G_{1}\right)\right)\left(M_{1}^{k}\left(G_{2}\right)+\bar{M}_{1}^{k}\left(G_{2}\right)\right)+\sum_{i=0}^{\alpha}\left(\begin{array}{c}
\alpha \\
i
\end{array}\right) \delta^{i}\left(S\left(G_{1}\right)\right)\left[M_{2}^{n-i}\left(S\left(G_{1}\right)\right)\left(M_{1}^{i}\left(G_{2}\right)+\bar{M}_{1}^{i}\left(G_{2}\right)\right)\right] \\
& +\alpha_{1}^{n-i}\left(M_{1}^{i}\left(G_{2}\right)+\bar{M}_{1}^{i}\left(G_{2}\right)\right)+2^{2 \alpha-1}\left(n_{2}^{2} e_{1}^{2}-n_{2} e_{1}\right), \\
\mathrm{UB}= & \sum_{\substack{m, k, l \\
m, k, l m+k+l=\alpha}} P_{m, k, l}\left[M_{1}^{2 m+k}\left(G_{1}\right) \bar{\chi}_{k}\left(G_{2}\right) \Delta\left(G_{2}\right)^{2 l}\right]+\Delta\left(G_{1}\right)^{2 m}\left[M_{1}^{2 l+k}\left(G_{2}\right)\left(\chi_{k}\left(G_{1}\right)+\bar{\chi}_{k}\left(G_{1}\right)\right)\right]+\Delta\left(G_{2}\right)^{2 l} \\
& \left(M_{1}^{k}\left(G_{1}\right)+\bar{M}_{1}^{k}\left(G_{1}\right)\right)\left(M_{1}^{k}\left(G_{2}\right)+\bar{M}_{1}^{k}\left(G_{2}\right)\right)+\sum_{i=0}^{\alpha}\left(\begin{array}{c}
\alpha \\
i
\end{array}\right) \Delta^{i}\left(S\left(G_{1}\right)\right)\left[M_{2}^{n-i}\left(S\left(G_{1}\right)\right)\left(M_{1}^{i}\left(G_{2}\right)+\bar{M}_{1}^{i}\left(G_{2}\right)\right)\right] \\
& +\alpha_{1}^{n-i}\left(M_{1}^{i}\left(G_{2}\right)+\bar{M}_{1}^{i}\left(G_{2}\right)\right)+2^{2 \alpha-1}\left(n_{2}^{2} e_{1}^{2}-n_{2} e_{1}\right) .
\end{aligned}
$$

Proof. Using equation (4), we have

Consider

$$
\bar{R}_{\alpha}\left(G_{1+S} G_{2}\right)=\sum A+\sum B+\sum C .
$$

$$
\begin{aligned}
\sum A & =\sum_{s_{1}, s_{2} \in V} \sum_{\left.s\left(G_{1}\right)-V\left(G_{1}\right)\right)} \sum_{x_{1}, x_{2} \in V_{G_{2}}}\left[d\left(s_{1}, x_{1}\right)\left(s_{2}, x_{2}\right)\right]^{\alpha} \\
& =\sum_{s_{1}, s_{2} \in V} \sum_{\left(S\left(G_{1}\right)-V\left(G_{1}\right)\right)}\left[d_{x_{1}, x_{2} \in V_{G_{2}}}\left(G_{S}\right)\left(s_{1}\right) d_{S\left(G_{1}\right)}\left(s_{2}\right)\right]^{\alpha}=\sum_{s_{1}, s_{2} \in V\left(S\left(G_{1}\right)-V\left(G_{1}\right)\right)} \sum_{x_{1}, x_{2} \in V_{G_{2}}}(2 \times 2)^{\alpha}, \\
\sum A & =2^{2 \alpha-1}\left(n_{2}^{2} e_{1}^{2}-n_{2} e_{1}\right), \\
\sum B & =\sum_{i=1}^{7} B_{i}, \\
\sum B_{1} & =\sum_{s \in V_{G_{1}}} \sum_{x_{1} x_{2} \notin E_{G_{2}}}\left[d\left(s, x_{1}\right) d\left(s, x_{2}\right)\right]^{\alpha}=\sum_{s \in V_{G_{1}}} \sum_{x_{1} x_{2} \notin E_{G_{2}}}\left[\left(d_{G_{1}}(s)+d_{G_{2}}\left(x_{1}\right)\right)\left(d_{G_{1}}(s)+d_{G_{2}}\left(x_{2}\right)\right)\right]^{\alpha} \\
& =\sum_{s \in V_{G_{1}}} \sum_{x_{1} x_{2} \notin E_{G_{2}}}\left[d_{G_{1}}^{2}(s)+d_{G_{1}}(s) d_{G_{2}}\left(x_{2}\right)+d_{G_{2}}\left(x_{1}\right)+d_{G_{2}}\left(x_{1}\right) d_{G_{2}}\left(x_{2}\right)\right]^{\alpha} .
\end{aligned}
$$

Using equation (6), 

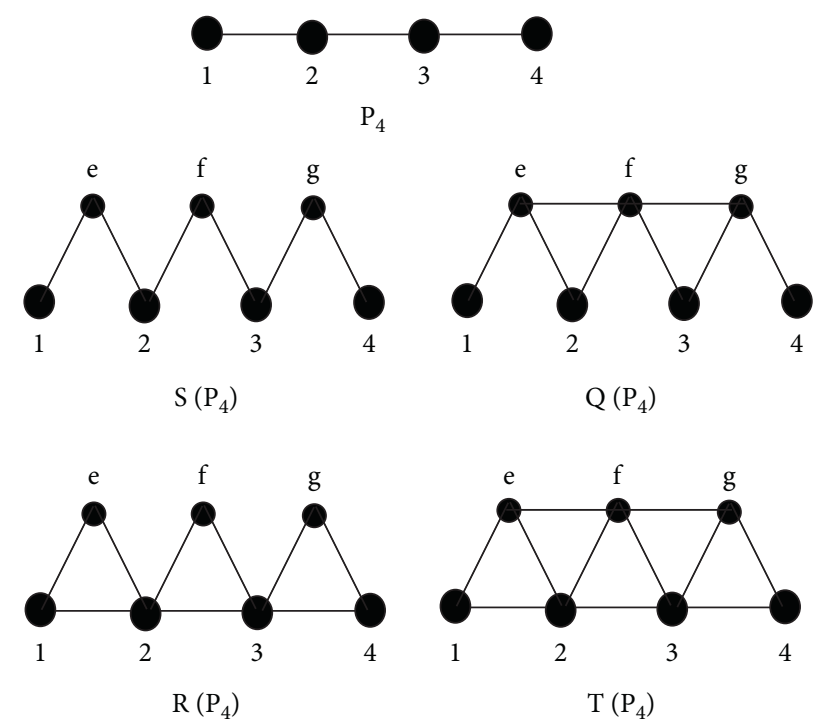

Figure 1: Graph $P_{4}$ and its subdivision.
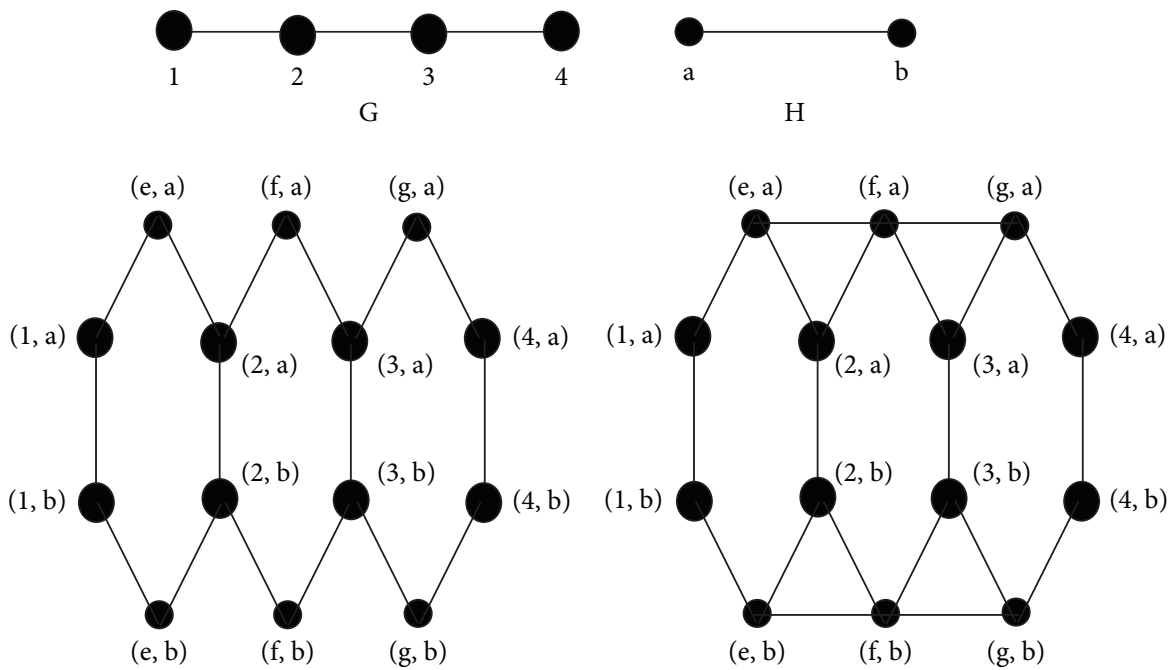

$\left(\mathrm{G}+{ }_{\mathrm{S}} \mathrm{H}\right)$

$\left(\mathrm{G}+{ }_{\mathrm{Q}} \mathrm{H}\right)$
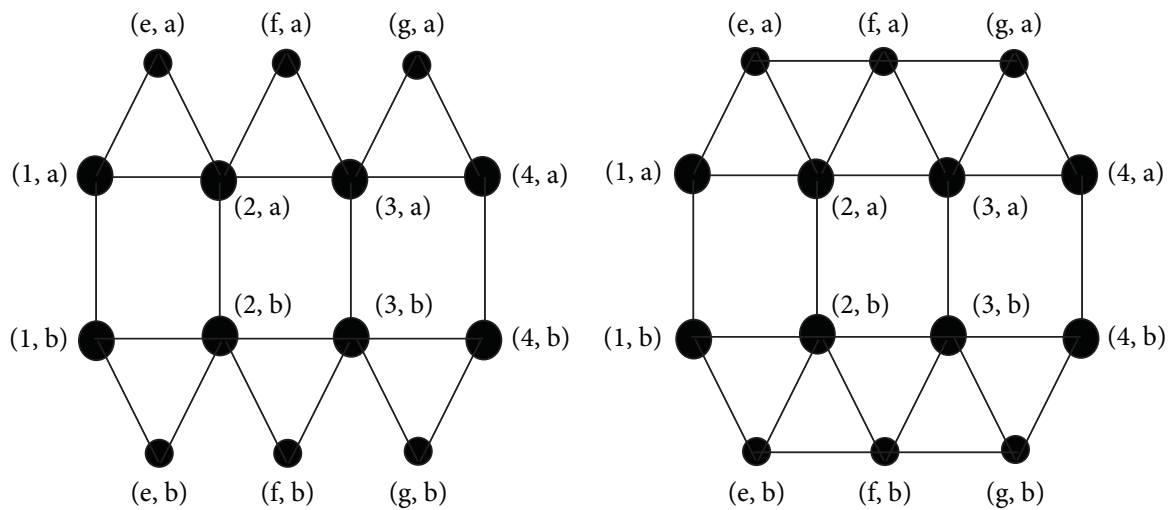

$\left(\mathrm{G}+{ }_{\mathrm{R}} \mathrm{H}\right)$

$\left(\mathrm{G}+_{\mathrm{T}} \mathrm{H}\right)$

Figure 2 : Graphs $G \cong P_{4}, H \cong P_{2}$, and $G_{+F} H \cong P_{4+F} P_{2}$. 


$$
\begin{aligned}
& =\sum_{s \in V_{G_{1}}} \sum_{x_{1} x_{2} \notin E_{G_{2}}}\left[\sum_{\substack{m, k, l \\
m, k, l m+k+l=\alpha}} P_{m, k, l} d_{G_{1}}^{2 m}(s) d_{G_{1}}^{k}(s)\left(d_{G_{2}}\left(x_{2}\right)+d_{G_{2}}\left(x_{1}\right)\right)^{k}\left(d_{G_{2}}\left(x_{1}\right) d_{G_{2}}\left(x_{2}\right)\right)^{l}\right] \\
& =\sum_{\substack{m, k, l \\
m, k, l m+k+l=\alpha}} P_{m, k, l}\left[\sum_{s \in V_{G_{1}}} \sum_{x_{1} x_{2} \notin E_{G_{2}}} d_{G_{1}}^{2 m+k}(s)\left(d_{G_{2}}\left(x_{2}\right)+d_{G_{2}}\left(x_{1}\right)\right)^{k}\left(d_{G_{2}}\left(x_{1}\right) d_{G_{2}}\left(x_{2}\right)\right)^{l}\right] .
\end{aligned}
$$

As we know, $\delta(G) \leq d(x) \leq \Delta(G), x \in V(G)$,

$$
\begin{aligned}
& \leq \sum_{m, k, l} P_{m, k, l}\left[M_{1}^{2 m+k}\left(G_{1}\right) \bar{\chi}_{k}\left(G_{2}\right) \Delta\left(G_{2}\right)^{2 l}\right], \\
\sum B_{2} & =\sum_{s_{1} s_{2} \in E_{G_{1}}} \sum_{x \in V_{G_{2}}}\left[d\left(s_{1}, x\right) d\left(s_{2}, x\right)\right]^{\alpha}=\sum_{s_{1} s_{2} \in E_{G_{1}}} \sum_{x \in V_{G_{2}}}\left[\left(d_{G_{1}}\left(s_{1}\right)+d_{G_{2}}(x)\right)\left(d_{G_{1}}\left(s_{1}\right)+d_{G_{2}}(x)\right)\right]^{\alpha} \\
& =\sum_{s_{1} s_{2} \in E_{G_{1}}} \sum_{x \in V_{G_{2}}}\left[d_{G_{1}}\left(s_{1}\right) d_{G_{1}}\left(s_{2}\right)+d_{G_{2}}(x)\left(d_{G_{1}}\left(s_{1}\right)+d_{G_{1}}\left(s_{2}\right)\right)+d_{G_{2}}(x) d_{G_{2}}(x)\right]^{\alpha} \\
& =\sum_{s_{1} s_{2} \in E_{G_{1}}} \sum_{x \in V_{G_{2}}}\left[\sum_{m, k, k, l} P_{m, k, l}\left(d_{G_{1}}\left(s_{1}\right) d_{G_{1}}\left(s_{2}\right)\right)^{m}\left(d_{G_{2}}(x)\left(d_{G_{1}}\left(s_{1}\right)+d_{G_{1}}\left(s_{2}\right)\right)\right)^{k}\left(d_{G_{2}}(x)\right)^{2 l}\right] \\
& =\sum_{m, k+l=\alpha} P_{m, k, l}\left[\sum_{s_{1} s_{2} \in E_{G_{1}}} \sum_{x \in V_{G_{2}}}\left(d_{G_{1}}\left(s_{1}\right) d_{G_{1}}\left(s_{2}\right)\right)^{m}\left(d_{G_{1}}\left(s_{1}\right)+d_{G_{1}}\left(s_{2}\right)\right)^{k}\left(d_{G_{2}}(x)\right)^{2 l+k}\right] \\
& \leq \sum_{m, k, l m+k+l=\alpha} P_{m, k, l}\left[M_{1}^{2 l+k}\left(G_{2}\right) \chi_{k}\left(G_{1}\right) \Delta\left(G_{1}\right)^{2 m}\right], \\
\sum B_{3} & =\sum_{s_{1} s_{2} \notin E_{G_{1}}} \sum_{x \in V_{G_{2}}}\left[d\left(s_{1}, x\right) d\left(s_{2}, x\right)\right]^{\alpha}=\sum_{s_{1} s_{2} \notin E_{G_{1}}} \sum_{x \in V_{G_{2}}}\left[\left(d_{G_{1}}\left(s_{1}\right)+d_{G_{2}}(x)\right)\left(d_{G_{1}}\left(s_{1}\right)+d_{G_{2}}(x)\right)\right]^{\alpha} \\
& =\sum_{s_{1} s_{2} \notin E_{G_{1}}} \sum_{x \in V_{G_{2}}}\left[d_{G_{1}}\left(s_{1}\right) d_{G_{1}}\left(s_{2}\right)+d_{G_{2}}(x)\left(d_{G_{1}}\left(s_{1}\right)+d_{G_{1}}\left(s_{2}\right)\right)+d_{G_{2}}(x) d_{G_{2}}(x)\right]^{\alpha} \\
& =\sum_{s_{1} s_{2} \notin E_{G_{1}}} \sum_{x \in V_{G_{2}}}\left[\left(d_{G_{1}}\left(s_{1}\right) d_{G_{1}}\left(s_{2}\right)\right)^{m}\left(d_{G_{2}}(x)\left(d_{G_{1}}\left(s_{1}\right)+d_{G_{1}}\left(s_{2}\right)\right)\right)^{k}\left(d_{G_{2}}(x)\right)^{2 l}\right] \\
& =\sum_{s_{1} s_{2} \notin E_{G_{1}}} \sum_{x \in V_{G_{2}}}\left[\sum_{m, k, l} P_{m, k, l}\left(d_{G_{1}}\left(s_{1}\right) d_{G_{1}}\left(s_{2}\right)\right)^{m}\left(d_{G_{1}}\left(s_{1}\right)+d_{G_{1}}\left(s_{2}\right)\right)^{k}\left(d_{G_{2}}(x)\right)^{2 l+k}\right] \\
& \sum
\end{aligned}
$$




$$
\begin{aligned}
& \leq \sum_{\substack{m, k, l \\
m, k, l m+k+l=\alpha}} P_{m, k, l}\left[M_{1}^{2 l+k}\left(G_{2}\right) \bar{\chi}_{k}\left(G_{1}\right) \Delta\left(G_{1}\right)^{2 m}\right], \\
& \sum B_{4}=\sum_{s_{1} s_{2} \in E_{G_{1}}} \sum_{x_{1} x_{2} \in E_{G_{2}}}\left[d\left(s_{1}, x_{1}\right) d\left(s_{2}, x_{2}\right)\right]^{\alpha} \\
& =\sum_{s_{1} s_{2} \in E_{G_{1}}} \sum_{x_{1} x_{2} \in E_{G_{2}}}\left[\left(d_{G_{1}}\left(s_{1}\right)+d_{G_{2}}\left(x_{1}\right)\right)\left(d_{G_{1}}\left(s_{2}\right)+d_{G_{2}}\left(x_{2}\right)\right)\right]^{\alpha} \\
& =\sum_{s_{1} s_{2} \in E_{G_{1}}} \sum_{x_{1} x_{2} \in E_{G_{2}}}\left[d_{G_{1}}\left(s_{1}\right) d_{G_{1}}\left(s_{2}\right)+d_{G_{1}}\left(s_{1}\right) d_{G_{2}}\left(x_{2}\right)+d_{G_{1}}\left(s_{2}\right) d_{G_{2}}\left(x_{1}\right)+d_{G_{2}}\left(x_{1}\right) d_{G_{2}}\left(x_{2}\right)\right]^{\alpha} \\
& =\sum_{\substack{m, k, l \\
m, k, l m+k+l=\alpha}} P_{m, k, l}\left[\sum_{s_{1} s_{2} \in E_{G_{1}}} \sum_{x_{1} x_{2} \in E_{G_{2}}}\left(d_{G_{1}}\left(s_{1}\right) d_{G_{1}}\left(s_{2}\right)\right)^{m}\left(d_{G_{1}}\left(s_{1}\right) d_{G_{2}}\left(x_{2}\right)+d_{G_{1}}\left(s_{2}\right) d_{G_{2}}\left(x_{1}\right)\right)^{k}\left(d_{G_{2}}\left(x_{1}\right) d_{G_{2}}\left(x_{2}\right)\right)^{l}\right] \\
& \leq \sum_{\substack{m, k, l \\
m, k, l m+k+l=\alpha}} P_{m, k, l}\left[\Delta\left(G_{1}\right)^{2 m} \Delta\left(G_{2}\right)^{2 l} M_{1}^{k}\left(G_{1}\right) M_{1}^{k}\left(G_{2}\right)\right] \\
& \sum B_{5}=\sum_{s_{1} s_{2} \notin E_{G_{1}}} \sum_{x_{1} x_{2} \in E_{G_{2}}}\left[d\left(s_{1}, x_{1}\right) d\left(s_{2}, x_{2}\right)\right]^{\alpha}, \\
& =\sum_{s_{1} s_{2} \notin E_{G_{1}}} \sum_{x_{1} x_{2} \in E_{G_{2}}}\left[\left(d_{G_{1}}\left(s_{1}\right)+d_{G_{2}}\left(x_{1}\right)\right)\left(d_{G_{1}}\left(s_{2}\right)+d_{G_{2}}\left(x_{2}\right)\right)\right]^{\alpha} \\
& =\sum_{s_{1} s_{2} \notin E_{G_{1}}} \sum_{x_{1} x_{2} \in E_{G_{2}}}\left[d_{G_{1}}\left(s_{1}\right) d_{G_{1}}\left(s_{2}\right)+d_{G_{1}}\left(s_{1}\right) d_{G_{2}}\left(x_{2}\right)+d_{G_{1}}\left(s_{2}\right) d_{G_{2}}\left(x_{1}\right)+d_{G_{2}}\left(x_{1}\right) d_{G_{2}}\left(x_{2}\right)\right]^{\alpha} \\
& =\sum_{s_{1} s_{2} \notin E_{G_{1}}} \sum_{x_{1} x_{2} \in E_{G_{2}}}\left[\sum_{\substack{m, k, l \\
m, k, l m+k+l=\alpha}} P_{m, k, l}\left(d_{G_{1}}\left(s_{1}\right) d_{G_{1}}\left(s_{2}\right)\right)^{m}\left(d_{G_{1}}\left(s_{1}\right) d_{G_{2}}\left(x_{2}\right)+d_{G_{1}}\left(s_{2}\right) d_{G_{2}}\left(x_{1}\right)\right)^{k}\left(d_{G_{2}}\left(x_{1}\right) d_{G_{2}}\left(x_{2}\right)\right)^{l}\right] \\
& \leq \sum_{\substack{m, k, l \\
m, k, l m+k+l=\alpha}} P_{m, k, l}\left[\Delta\left(G_{1}\right)^{2 m} \Delta\left(G_{2}\right)^{2 l} \bar{M}_{1}^{k}\left(G_{1}\right) M_{1}^{k}\left(G_{2}\right)\right], \\
& \sum B_{6}=\sum_{s_{1} s_{2} \in E_{G_{1}}} \sum_{x_{1} x_{2} \notin E_{G_{2}}}\left[d\left(s_{1}, x_{1}\right) d\left(s_{2}, x_{2}\right)\right]^{\alpha} \\
& =\sum_{s_{1} s_{2} \notin E_{G_{1}}} \sum_{x_{1} x_{2} \in E_{G_{2}}}\left[\left(d_{G_{1}}\left(s_{1}\right)+d_{G_{2}}\left(x_{1}\right)\right)\left(d_{G_{1}}\left(s_{2}\right)+d_{G_{2}}\left(x_{2}\right)\right)\right]^{\alpha} \\
& =\sum_{s_{1} s_{2} \notin E_{G_{1}}} \sum_{x_{1} x_{2} \notin E_{G_{2}}}\left[d_{G_{1}}\left(s_{1}\right) d_{G_{1}}\left(s_{2}\right)+d_{G_{1}}\left(s_{1}\right) d_{G_{2}}\left(x_{2}\right)+d_{G_{1}}\left(s_{2}\right) d_{G_{2}}\left(x_{1}\right)+d_{G_{2}}\left(x_{1}\right) d_{G_{2}}\left(x_{2}\right)\right]^{\alpha} \\
& =\sum_{s_{1} s_{2} \notin E_{G_{1}}} \sum_{x_{1} x_{2} \notin E_{G_{2}}}\left[\sum_{\substack{m, k, l \\
m, k, l m+k+l=\alpha}} P_{m, k, l}\left(d_{G_{1}}\left(s_{1}\right) d_{G_{1}}\left(s_{2}\right)\right)^{m}\left(d_{G_{1}}\left(s_{1}\right) d_{G_{2}}\left(x_{2}\right)+d_{G_{1}}\left(s_{2}\right) d_{G_{2}}\left(x_{1}\right)\right)^{k}\left(d_{G_{2}}\left(x_{1}\right) d_{G_{2}}\left(x_{2}\right)\right)^{l}\right] \\
& \leq \sum_{\substack{m, k, l \\
m, k, l m+k+l=\alpha}} P_{m, k, l}\left[\Delta\left(G_{1}\right)^{2 m} \Delta\left(G_{2}\right)^{2 l} M_{1}^{k}\left(G_{1}\right) \bar{M}_{1}^{k}\left(G_{2}\right)\right]
\end{aligned}
$$




$$
\begin{aligned}
& \sum B_{7}=\sum_{s_{1} s_{2} \in E_{G_{1}}} \sum_{x_{1} x_{2} \in E_{G_{2}}}\left[d\left(s_{1}, x_{1}\right) d\left(s_{2}, x_{2}\right)\right]^{\alpha} \\
& =\sum_{s_{1} s_{2} \notin E_{G_{1}}} \sum_{x_{1} x_{2} \in E_{G_{2}}}\left[\left(d_{G_{1}}\left(s_{1}\right)+d_{G_{2}}\left(x_{1}\right)\right)\left(d_{G_{1}}\left(s_{2}\right)+d_{G_{2}}\left(x_{2}\right)\right)\right]^{\alpha} \\
& =\sum_{s_{1} s_{2} \notin E_{G_{1}}} \sum_{x_{1} x_{2} \notin E_{G_{2}}}\left[d_{G_{1}}\left(s_{1}\right) d_{G_{1}}\left(s_{2}\right)+d_{G_{1}}\left(s_{1}\right) d_{G_{2}}\left(x_{2}\right)+d_{G_{1}}\left(s_{2}\right) d_{G_{2}}\left(x_{1}\right)+d_{G_{2}}\left(x_{1}\right) d_{G_{2}}\left(x_{2}\right)\right]^{\alpha} \\
& =\sum_{s_{1} s_{2} \notin E_{G_{1}}} \sum_{x_{1} x_{2} \notin E_{G_{2}}}\left[\sum_{\substack{m, k, l \\
m, k, l m+k+l=\alpha}} P_{m, k, l}\left(d_{G_{1}}\left(s_{1}\right) d_{G_{1}}\left(s_{2}\right)\right)^{m}\left(d_{G_{1}}\left(s_{1}\right) d_{G_{2}}\left(x_{2}\right)+d_{G_{1}}\left(s_{2}\right) d_{G_{2}}\left(x_{1}\right)\right)^{k}\left(d_{G_{2}}\left(x_{1}\right) d_{G_{2}}\left(x_{2}\right)\right)^{l}\right] \\
& \leq \sum_{\substack{m, k, l \\
m, k, l m+k+l=\alpha}} P_{m, k, l}\left[\Delta\left(G_{1}\right)^{2 m} \Delta\left(G_{2}\right)^{2 l} \bar{M}_{1}^{k}\left(G_{1}\right) \bar{M}_{1}^{k}\left(G_{2}\right)\right], \\
& \sum B \leq \sum_{\substack{m, k, l \\
m, k, l m+k+l=\alpha}} P_{m, k, l}\left[M_{1}^{2 m+k}\left(G_{1}\right) \bar{\chi}_{k}\left(G_{2}\right) \Delta\left(G_{2}\right)^{2 l}\right]+\Delta\left(G_{1}\right)^{2 m}\left[M_{1}^{2 l+k}\left(G_{2}\right)\left(\chi_{k}\left(G_{1}\right)+\bar{\chi}_{k}\left(G_{1}\right)\right)\right] \\
& +\Delta\left(G_{2}\right)^{2 l}\left(M_{1}^{k}\left(G_{1}\right)+\bar{M}_{1}^{k}\left(G_{1}\right)\right)\left(M_{1}^{k}\left(G_{2}\right)+\bar{M}_{1}^{k}\left(G_{2}\right)\right), \\
& \sum C=\sum_{i=1}^{5} C_{i} \\
& \sum C_{1}=\sum_{s_{1} s_{2} \in E\left(s\left(G_{1}\right)\right)} \sum_{x_{1}, x_{2} \in V_{G_{2}}}\left[d\left(s_{1}, x\right) d\left(s_{2}, x\right)\right]^{\alpha}
\end{aligned}
$$

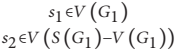

$$
\begin{aligned}
& =\sum_{\substack{s_{1} s_{2} \in E\left(S\left(G_{1}\right)\right) \\
s_{1} \in V\left(G_{1}\right)}} \sum_{x_{1}, x_{2} \in V_{G_{2}}}\left[\left(d_{S}\left(s_{1}\right)+d_{G_{2}}\left(x_{1}\right)\right)\left(d_{S}\left(s_{2}\right)\right)\right]^{\alpha} \\
& \begin{array}{c}
s_{1} \in V\left(G_{1}\right) \\
s_{2} \in V\left(S\left(G_{1}\right)-V\left(G_{1}\right)\right)
\end{array}
\end{aligned}
$$

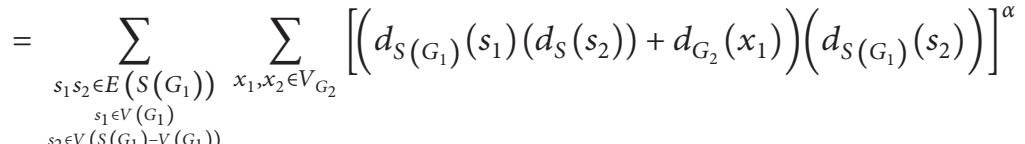

$$
\begin{aligned}
& =\sum_{\substack{s_{1} s_{2} \in E\left(S\left(G_{1}\right)\right) \\
\text { s } \\
s_{2} \in V\left(G_{1}\right)}} \sum_{\substack{\left.\left.x_{1}, x_{2} \in V_{G_{1}}\right)-V\left(G_{1}\right)\right) \\
G_{G_{2}}}}\left[\sum_{i=0}^{\alpha}\left(\begin{array}{c}
\alpha \\
i
\end{array}\right)\left(d_{S\left(G_{1}\right)}\left(s_{1}\right)\left(d_{S}\left(s_{2}\right)\right)^{n-i} d_{G_{2}}\left(x_{1}\right)\right)^{i}\left(d_{S\left(G_{1}\right)}\left(s_{2}\right)\right)^{i}\right] \\
& \leq \sum_{i=0}^{\alpha}\left(\begin{array}{c}
\alpha \\
i
\end{array}\right)\left[M_{2}^{n-i}\left(S\left(G_{1}\right) M_{1}^{i}\left(G_{2}\right) \Delta^{i} S\left(G_{1}\right)\right)\right], \\
& \sum C_{2}=\sum_{\substack{s_{1} s_{2} \in E\left(S\left(G_{1}\right)\right) \\
s_{1} \in V\left(G_{1}\right)}} \sum_{\substack{1 \\
x_{2} \\
x_{2} \notin V}}\left[d\left(s_{1}, x\right) d\left(s_{2}, x\right)\right]^{\alpha} \\
& s_{2} \in V\left(s\left(G_{1}\right)-V\left(G_{1}\right)\right) \\
& =\sum_{\substack{s_{1} s_{2} \in E\left(s\left(G_{1}\right)\right) \\
\text { s } \in V V\left(G_{1}\right) \\
s_{2} \notin V\left(S\left(G_{1}\right)-V\left(G_{1}\right)\right)}} \sum_{\substack{1 \\
1 \\
x_{2} \notin V_{G_{2}}}}\left[\left(d_{S}\left(s_{1}\right)+d_{G_{2}}\left(x_{1}\right)\right)\left(d_{S}\left(s_{2}\right)\right)\right]^{\alpha}
\end{aligned}
$$


8

Journal of Mathematics

$$
\begin{aligned}
& =\sum_{\substack{s_{1} s_{2} \in E\left(S\left(G_{1}\right)\right) \\
s_{1} \in V\left(G_{1}\right) \\
s_{2} \in V\left(S\left(G_{1}\right)-V\left(G_{1}\right)\right)}} \sum_{x_{1}, x_{2} \notin V_{G_{2}}}\left[\left(d_{S\left(G_{1}\right)}\left(s_{1}\right)\left(d_{S}\left(s_{2}\right)\right)+d_{G_{2}}\left(x_{1}\right)\right)\left(d_{S\left(G_{1}\right)}\left(s_{2}\right)\right)\right]^{\alpha} \\
& =\sum_{\substack{s_{1} s_{2} \in E\left(S\left(G_{1}\right)\right) \\
\text { s } \in V\left(G_{1}\right) \\
s_{2} \in V\left(S\left(G_{1}\right)-V\left(G_{1}\right)\right)}} \sum_{x_{1}, x_{2} \notin V_{G_{2}}}\left[\sum_{i=0}^{\alpha}\left(\begin{array}{c}
\alpha \\
i
\end{array}\right)\left(d_{S\left(G_{1}\right)}\left(s_{1}\right)\left(d_{S}\left(s_{2}\right)\right)^{n-i} d_{G_{2}}\left(x_{1}\right)\right)^{i}\left(d_{S\left(G_{1}\right)}\left(s_{2}\right)\right)^{i}\right] \\
& \leq \sum_{i=0}^{\alpha}\left(\begin{array}{c}
\alpha \\
i
\end{array}\right)\left[M_{2}^{n-i}\left(S\left(G_{1}\right)\right) \bar{M}_{1}^{i}\left(G_{2}\right) \Delta^{i}\left(S\left(G_{1}\right)\right)\right] \text {, } \\
& \sum C_{3}=\sum_{s_{1} s_{2} \notin E\left(S\left(G_{1}\right)\right)} \sum_{x \in V_{G_{2}}}\left[d\left(s_{1}, x\right) d\left(s_{2}, x\right)\right]^{\alpha} \\
& \begin{array}{c}
s_{1} \in V\left(G_{1}\right) \\
s_{2} \in V\left(S\left(G_{1}\right)-V\left(G_{1}\right)\right)
\end{array} \\
& =\sum_{s_{1} s_{2} \notin E\left(S\left(G_{1}\right)\right)} \sum_{x \in V_{G_{2}}}\left[\left(d_{S}\left(s_{1}\right)+d_{G_{2}}(x)\right)\left(d_{S}\left(s_{2}\right)\right)\right]^{\alpha} \\
& \begin{array}{c}
s_{1} \in V\left(G_{1}\right) \\
s_{2} \in V\left(S\left(G_{1}\right)-V\left(G_{1}\right)\right)
\end{array} \\
& =\sum_{\substack{s_{1} s_{2} \notin E\left(S\left(G_{1}\right)\right) \\
\text { site }\left(G_{1}\right) \\
s_{2} \in V\left(S\left(G_{1}\right)-V\left(G_{1}\right)\right)}} \sum_{x \in V_{G_{2}}}\left[\left(d_{S\left(G_{1}\right)}\left(s_{1}\right)\left(d_{S}\left(s_{2}\right)\right)+d_{G_{2}}(x)\right)\left(d_{S\left(G_{1}\right)}\left(s_{2}\right)\right)\right]^{\alpha} \\
& =\sum_{\substack{s_{1} s_{2} \notin E\left(S\left(G_{1}\right)\right) \\
\text { s } \in V\left(G_{1}\right) \\
s_{2} \in V\left(S\left(G_{1}\right)-V\left(G_{1}\right)\right)}} \sum_{x \in V_{G_{2}}}\left[\sum_{i=0}^{\alpha}\left(\begin{array}{c}
\alpha \\
i
\end{array}\right)\left(d_{S\left(G_{1}\right)}\left(s_{1}\right)\left(d_{S}\left(s_{2}\right)\right)\right)^{n-i}\left(d_{G_{2}}(x)\right)^{i}\left(d_{S\left(G_{1}\right)}\left(s_{2}\right)\right)^{i}\right] \\
& \leq \sum_{i=0}^{\alpha}\left(\begin{array}{c}
\alpha \\
i
\end{array}\right) \alpha_{1}^{n-i}\left[M_{1}^{i}\left(G_{2}\right) \Delta^{i}\left(S\left(G_{1}\right)\right)\right] \\
& \sum C_{4}=\sum_{\substack{s_{1} s_{2} \notin E\left(S\left(G_{1}\right)\right) \\
s_{1} \in V\left(G_{1}\right) \\
s_{2} \in V\left(S\left(G_{1}\right)-V\left(G_{1}\right)\right)}} \sum_{x_{1}, x_{2} \in V_{G_{2}}}\left[d\left(s_{1}, x\right) d\left(s_{2}, x\right)\right]^{\alpha} \\
& =\sum_{\substack{s_{1} s_{2} \notin E\left(S\left(G_{1}\right)\right) \\
s_{1} \in V\left(G_{1}\right)}} \sum_{x_{1}, x_{2} \in V_{G_{2}}}\left[\left(d_{S}\left(s_{1}\right)+d_{G_{2}}\left(x_{1}\right)\right)\left(d_{S}\left(s_{2}\right)\right)\right]^{\alpha} \\
& s_{2} \in V\left(S\left(G_{1}\right)-V\left(G_{1}\right)\right) \\
& =\sum_{\substack{s_{1} s_{2} \notin E\left(S\left(G_{1}\right)\right) \\
\text { s } \in V\left(G_{1}\right) \\
s_{2} \in V\left(S\left(G_{1}\right)-V\left(G_{1}\right)\right)}} \sum_{x_{1}, x_{2} \in V_{G_{2}}}\left[\left(d_{S\left(G_{1}\right)}\left(s_{1}\right)\left(d_{S}\left(s_{2}\right)\right)+d_{G_{2}}\left(x_{1}\right)\right)\left(d_{S\left(G_{1}\right)}\left(s_{2}\right)\right)\right]^{\alpha} \\
& =\sum_{\substack{s_{1} s_{2} \notin E\left(S\left(G_{1}\right)\right) \\
\text { s } \in\left(G_{1}\right) \\
s_{2} \in V\left(S\left(G_{1}\right)-V\left(G_{1}\right)\right)}} \sum_{x_{1}, x_{2} \in V_{G_{2}}}\left[\sum_{i=0}^{\alpha}\left(\begin{array}{c}
\alpha \\
i
\end{array}\right)\left(d_{S\left(G_{1}\right)}\left(s_{1}\right)\left(d_{S}\left(s_{2}\right)\right)^{n-i} d_{G_{2}}\left(x_{1}\right)\right)^{i}\left(d_{S\left(G_{1}\right)}\left(s_{2}\right)\right)^{i}\right] \\
& \leq \sum_{i=0}^{\alpha}\left(\begin{array}{c}
\alpha \\
i
\end{array}\right)\left[\alpha_{1}^{n-i} M_{1}^{i}\left(G_{2}\right) \Delta^{i}\left(S\left(G_{1}\right)\right)\right]
\end{aligned}
$$




$$
\begin{aligned}
& \sum C_{5}=\sum_{\substack{s_{1} s_{2} \notin E\left(s\left(G_{1}\right)\right) \\
s_{1} \in V\left(G_{1}\right) \\
s_{2} \in V\left(s\left(G_{1}\right)-V\left(G_{1}\right)\right)}} \sum_{\substack{1 \\
1 \\
x_{2} \notin V_{G_{2}}}}\left[d\left(s_{1}, x\right) d\left(s_{2}, x\right)\right]^{\alpha} \\
& =\sum_{\substack{s_{1} s_{2} \notin E\left(s\left(G_{1}\right)\right) \\
\text { s.teV (G) } \\
s_{2} \notin V\left(S\left(G_{1}\right)-V\left(G_{1}\right)\right)}} \sum_{x_{1}, x_{2} \in V_{G_{2}}}\left[\left(d_{S}\left(s_{1}\right)+d_{G_{2}}\left(x_{1}\right)\right)\left(d_{S}\left(s_{2}\right)\right)\right]^{\alpha} \\
& =\sum_{\substack{s_{1} s_{2} \notin E\left(S\left(G_{1}\right)\right) \\
s_{1} \in V\left(G_{1}\right) \\
s_{2} \in V\left(S\left(G_{1}\right)-V\left(G_{1}\right)\right)}} \sum_{\substack{x_{1}, x_{2} \notin V_{G_{2}} \\
s_{1}}}\left[\left(d_{S\left(G_{1}\right)}\left(s_{1}\right)\left(d_{S}\left(s_{2}\right)\right)+d_{G_{2}}\left(x_{1}\right)\right)\left(d_{S\left(G_{1}\right)}\left(s_{2}\right)\right)\right]^{\alpha} \\
& =\sum_{\substack{s_{1} s_{2} \notin E\left(S\left(G_{1}\right)\right) \\
\text { s } \in V\left(G_{1}\right) \\
s_{2} \in V\left(S\left(G_{1}\right)-V\left(G_{1}\right)\right)}} \sum_{x_{1}, x_{2} \notin V_{G_{2}}}\left[\sum_{i=0}^{\alpha}\left(\begin{array}{c}
\alpha \\
i
\end{array}\right)\left(d_{S\left(G_{1}\right)}\left(s_{1}\right)\left(d_{S}\left(s_{2}\right)\right)^{n-i} d_{G_{2}}\left(x_{1}\right)\right)^{i}\left(d_{S\left(G_{1}\right)}\left(s_{2}\right)\right)^{i}\right] \\
& \leq \sum_{i=0}^{\alpha}\left(\begin{array}{c}
\alpha \\
i
\end{array}\right)\left[\alpha_{1}^{n-i} \bar{M}_{1}^{i}\left(G_{2}\right) \Delta^{i}\left(S\left(G_{1}\right)\right)\right] .
\end{aligned}
$$

We obtained upper bound by putting value of $\sum A, \sum B$, and $\sum C$ in equation (13). Similarly, lower bound can be obtained using smallest degree of graphs $G_{1}$ and $G_{2}$.

$$
\mathrm{LB} \leq \bar{R}_{\alpha}\left(G_{1+R} G_{2}\right) \leq \mathrm{UB},
$$

Theorem 2. Let $G_{1+R} G_{2}$ be a R-sum graph, then its general Randic coindex $\bar{R}_{\alpha}\left(G_{1+R} G_{2}\right)$ is given as:

$$
\begin{aligned}
\mathrm{LB}= & \sum_{\substack{m, k, l \\
m, k, l m+k+l=\alpha}} P_{m, k, l} 2^{2 m+k}\left[M_{1}^{2 m+k}\left(G_{1}\right) \bar{\chi}_{k}\left(G_{2}\right) \delta\left(G_{2}\right)^{2 l}\right]+\delta\left(G_{1}\right)^{2 m} \\
& {\left[M_{1}^{2 l+k}\left(G_{2}\right)\left(\chi_{k}\left(G_{1}\right)+\bar{\chi}_{k}\left(G_{1}\right)\right)\right]+\delta\left(G_{2}\right)^{2 l}\left(M_{1}^{k}\left(G_{1}\right)+\bar{M}_{1}^{k}\left(G_{1}\right)\right)\left(M_{1}^{k}\left(G_{2}\right)+\bar{M}_{1}^{k}\left(G_{2}\right)\right) } \\
& +\sum_{i=0}^{\alpha}\left(\begin{array}{c}
\alpha \\
i
\end{array}\right) \delta^{i}\left(S\left(G_{1}\right)\right)\left[M_{2}^{n-i}\left(S\left(G_{1}\right)\right)\left(M_{1}^{i}\left(G_{2}\right)+\bar{M}_{1}^{i}\left(G_{2}\right)\right)\right] \\
& +\alpha_{1}^{n-i}\left(M_{1}^{i}\left(G_{2}\right)+\bar{M}_{1}^{i}\left(G_{2}\right)\right)+2^{2 \alpha-1}\left(n_{2}^{2} e_{1}^{2}-n_{2} e_{1}\right), \\
\mathrm{UB}= & \sum_{m, k, l} P_{m, k, l} 2^{2 m+k}\left[M_{1}^{2 m+k}\left(G_{1}\right) \bar{\chi}_{k}\left(G_{2}\right) \Delta\left(G_{2}\right)^{2 l}\right]+\Delta\left(G_{1}\right)^{2 m} \\
& m, k, l m+k+l=\alpha \\
& {\left[M_{1}^{2 l+k}\left(G_{2}\right)\left(\chi_{k}\left(G_{1}\right)+\bar{\chi}_{k}\left(G_{1}\right)\right)\right]+\Delta\left(G_{2}\right)^{2 l}\left(M_{1}^{k}\left(G_{1}\right)+\bar{M}_{1}^{k}\left(G_{1}\right)\right)\left(M_{1}^{k}\left(G_{2}\right)+\bar{M}_{1}^{k}\left(G_{2}\right)\right) } \\
& +\sum_{i=0}^{\alpha}\left(\begin{array}{c}
\alpha \\
i
\end{array}\right) \Delta^{i}\left(S\left(G_{1}\right)\right)\left[M_{2}^{n-i}\left(S\left(G_{1}\right)\right)\left(M_{1}^{i}\left(G_{2}\right)+\bar{M}_{1}^{i}\left(G_{2}\right)\right)\right]+\alpha_{1}^{n-i}\left(M_{1}^{i}\left(G_{2}\right)+\bar{M}_{1}^{i}\left(G_{2}\right)\right)+2^{2 \alpha-1}\left(n_{2}^{2} e_{1}^{2}-n_{2} e_{1}\right) . \\
\text { Proof. Using equation }(4), \text { we have } & \bar{R}_{\alpha}\left(G_{1+R} G_{2}\right)=\sum A+\sum B+\sum C .
\end{aligned}
$$


The value of $\sum A$ follows from equation (11),

$$
\begin{aligned}
& \sum A=2^{2 \alpha-1}\left(n_{2}^{2} e_{1}^{2}-n_{2} e_{1}\right), \\
& \sum B=\sum_{i=1}^{7} B_{i} \\
& \sum B_{1}=\sum_{s \in V_{G_{1}}} \sum_{x_{1} x_{2} \notin E_{G_{2}}}\left[d\left(s, x_{1}\right) d\left(s, x_{2}\right)\right]^{\alpha}=\sum_{s \in V_{G_{1}}} \sum_{x_{1} x_{2} \notin E_{G_{2}}}\left[\left(d_{R\left(G_{1}\right)}(s)+d_{G_{2}}\left(x_{1}\right)\right)\left(d_{R\left(G_{1}\right)}(s)+d_{G_{2}}\left(x_{2}\right)\right)\right]^{\alpha} \\
& =\sum_{s \in V_{G_{1}}} \sum_{x_{1} x_{2} \notin E_{G_{2}}}\left[4 d_{G_{1}}(s) d_{G_{1}}(s)+2 d_{G_{1}}(s) d_{G_{2}}\left(x_{2}\right)+d_{G_{1}}(s) d_{G_{2}}\left(x_{1}\right)+d_{G_{2}}\left(x_{1}\right) d_{G_{2}}\left(x_{2}\right)\right]^{\alpha} \\
& =\sum_{s \in V_{G_{1}}} \sum_{x_{1} x_{2} \notin E_{G_{2}}}\left[4 d_{G_{1}}^{2}(s)+2 d_{G_{1}}(s) d_{G_{2}}\left(x_{2}\right)+d_{G_{2}}\left(x_{1}\right)+d_{G_{2}}\left(x_{1}\right) d_{G_{2}}\left(x_{2}\right)\right]^{\alpha} \\
& =\sum_{s \in V_{G_{1}}} \sum_{x_{1} x_{2} \notin E_{G_{2}}}\left[\sum_{\substack{m, k, l \\
m, k, l m+k+l=\alpha}} P_{m, k, l} 4^{m} d_{G_{1}}^{2 m}(s) 2_{k} d_{G_{1}}^{k}(s)\left(d_{G_{2}}\left(x_{2}\right)\right)+d_{G_{2}}\left(x_{1}\right)^{k}\left(d_{G_{2}}\left(x_{1}\right) d_{G_{2}}\left(x_{2}\right)\right)^{l}\right] \\
& =\sum_{s \in V_{G_{1}}} \sum_{x_{1} x_{2} \notin E_{G_{2}}}\left[\sum_{\substack{m, k, l \\
m, k, l m+k+l=\alpha}} P_{m, k, l^{2}}{ }^{2 m+k} d_{G_{1}}^{2 m+k}(s)\left(d_{G_{2}}\left(x_{2}\right)\right)+d_{G_{2}}\left(x_{1}\right)^{k}\left(d_{G_{2}}\left(x_{1}\right) d_{G_{2}}\left(x_{2}\right)\right)^{l}\right] \\
& \leq 2^{2 m+k} \sum_{\substack{m, k, l \\
m, k, l m+k+l=\alpha}} P_{m, k, l}\left[M_{1}^{2 m+k}\left(G_{1}\right) \bar{\chi}_{k}\left(G_{2}\right) \Delta\left(G_{2}\right)^{2 l}\right], \\
& \sum B_{2}=\sum_{s_{1} s_{2} \in E_{G_{1}}} \sum_{x \in V_{G_{2}}}\left[d\left(s_{1}, x\right) d\left(s_{2}, x\right)\right]^{\alpha}=\sum_{s_{1} s_{2} \in E_{G_{1}}} \sum_{x \in V_{G_{2}}}\left[\left(d_{R\left(G_{1}\right)}\left(s_{1}\right)+d_{G_{2}}(x)\right)\left(d_{R\left(G_{1}\right)}\left(s_{1}\right)+d_{G_{2}}(x)\right)\right]^{\alpha} \\
& =\sum_{s_{1} s_{2} \in E_{G_{1}}} \sum_{x \in V_{G_{2}}}\left[d_{R\left(G_{1}\right)}\left(s_{1}\right) d_{R\left(G_{1}\right)}\left(s_{2}\right)+d_{G_{2}}(x)\left(d_{R\left(G_{1}\right)}\left(s_{1}\right)+d_{R\left(G_{1}\right)}\left(s_{2}\right)\right)+d_{G_{2}}(x) d_{G_{2}}(x)\right]^{\alpha} \\
& =\sum_{s_{1} s_{2} \in E_{G_{1}}} \sum_{x \in V_{G_{2}}}\left[\left(\sum_{\substack{m, k, l \\
m, k, l m+k+l=\alpha}} P_{m, k, l} 4 d_{G_{1}}\left(s_{1}\right) d_{G_{1}}\left(s_{2}\right)\right)^{m}\left(2 d_{G_{2}}(x)\left(d_{G_{1}}\left(s_{1}\right)+d_{G_{1}}\left(s_{2}\right)\right)\right)^{k}\left(d_{G_{2}}(x)\right)^{2 l}\right] \\
& =2^{2 m+k} \sum_{s_{1} s_{2} \in E_{G_{1}}} \sum_{x \in V_{G_{2}}}\left[\sum_{\substack{m, k, l \\
m, k, l m+k+l=\alpha}} P_{m, k, l}\left(d_{G_{1}}\left(s_{1}\right) d_{G_{1}}\left(s_{2}\right)\right)^{m}\left(d_{G_{1}}\left(s_{1}\right)+d_{G_{1}}\left(s_{2}\right)\right)^{k}\left(d_{G_{2}}(x)\right)^{2 l+k}\right] \\
& \leq 2^{2 m+k} \sum_{\substack{m, k, l \\
m, k, l m+k+l=\alpha}} P_{m, k, l}\left[M_{1}^{2 l+k}\left(G_{2}\right) \chi_{k}\left(G_{1}\right) \Delta\left(G_{1}\right)^{2 m}\right], \\
& \sum B_{3}=\sum_{s_{1} s_{2} \notin E_{G_{1}}} \sum_{x \in V_{G_{2}}}\left[d\left(s_{1}, x\right) d\left(s_{2}, x\right)\right]^{\alpha}=\sum_{s_{1} s_{2} \in E_{G_{1}}} \sum_{x \notin V_{G_{2}}}\left[\left(d_{R\left(G_{1}\right)}\left(s_{1}\right)+d_{G_{2}}(x)\right)\left(d_{R\left(G_{1}\right)}\left(s_{1}\right)+d_{G_{2}}(x)\right)\right]^{\alpha} \\
& =\sum_{s_{1} s_{2} \notin E_{G_{1}}} \sum_{x \in V_{G_{2}}}\left[d_{R\left(G_{1}\right)}\left(s_{1}\right) d_{R\left(G_{1}\right)}\left(s_{2}\right)+d_{G_{2}}(x)\left(d_{R\left(G_{1}\right)}\left(s_{1}\right)+d_{R\left(G_{1}\right)}\left(s_{2}\right)\right)+d_{G_{2}}(x) d_{G_{2}}(x)\right]^{\alpha}
\end{aligned}
$$




$$
\begin{aligned}
& =\sum_{s_{1} s_{2} \notin E_{G_{1}}} \sum_{x \in V_{G_{2}}}\left[\sum_{\substack{m, k, l \\
m, k, l m+k+l=\alpha}} P_{m, k, l}\left(4 d_{G_{1}}\left(s_{1}\right) d_{G_{1}}\left(s_{2}\right)\right)^{m}\left(2 d_{G_{2}}(x)\left(d_{G_{1}}\left(s_{1}\right)+d_{G_{1}}\left(s_{2}\right)\right)^{k}\left(d_{G_{2}}(x)\right)^{2 l}\right]\right. \\
& =2^{2 m+k} \sum_{s_{1} s_{2} \notin E_{G_{1}}} \sum_{x \in V_{G_{2}}}\left[\sum_{\substack{m, k, l \\
m, k, l m+k+l=\alpha}} P_{m, k, l}\left(d_{G_{1}}\left(s_{1}\right) d_{G_{1}}\left(s_{2}\right)\right)^{m}\left(d_{G_{1}}\left(s_{1}\right)+d_{G_{1}}\left(s_{2}\right)\right)^{k}\left(d_{G_{2}}(x)\right)^{2 l+k}\right] \\
& \leq 2^{2 m+k} \sum_{\substack{m, k, l \\
m, k, l m+k+l=\alpha}} P_{m, k, l}\left[M_{1}^{2 l+k}\left(G_{2}\right) \bar{\chi}_{k}\left(G_{1}\right) \Delta\left(G_{1}\right)^{2 m}\right], \\
& \sum B_{4}=\sum_{s_{1} s_{2} \in E_{G_{1}}} \sum_{x_{1} x_{2} \in E_{G_{2}}}\left[d\left(s_{1}, x_{1}\right) d\left(s_{2}, x_{2}\right)\right]^{\alpha} \\
& =\sum_{s_{1} s_{2} \in E_{G_{1}}} \sum_{x_{1} x_{2} \in E_{G_{2}}}\left[\left(d_{R\left(G_{1}\right)}\left(s_{1}\right)+d_{G_{2}}\left(x_{1}\right)\right)\left(d_{R\left(G_{1}\right)}\left(s_{2}\right)+d_{G_{2}}\left(x_{2}\right)\right)\right]^{\alpha} \\
& =\sum_{s_{1} s_{2} \in E_{G_{1}}} \sum_{x_{1} x_{2} \in E_{G_{2}}}\left[d_{R\left(G_{1}\right)}\left(s_{1}\right) d_{R\left(G_{1}\right)}\left(s_{2}\right)+d_{R\left(G_{1}\right)}\left(s_{1}\right) d_{G_{2}}\left(x_{2}\right)+d_{R\left(G_{1}\right)}\left(s_{2}\right) d_{G_{2}}\left(x_{1}\right)+d_{G_{2}}\left(x_{1}\right) d_{G_{2}}\left(x_{2}\right)\right]^{\alpha} \\
& =\sum_{s_{1} s_{2} \in E_{G_{1}}} \sum_{x_{1} x_{2} \in E_{G_{2}}}\left[\sum_{\substack{m, k, l \\
m, k, l m+k+l=\alpha}} P_{m, k, l}\left(4 d_{G_{1}}\left(s_{1}\right) d_{G_{1}}\left(s_{2}\right)\right)^{m}\left(2 d_{G_{1}}\left(s_{1}\right) d_{G_{2}}\left(x_{2}\right)+d_{G_{1}}\left(s_{2}\right) d_{G_{2}}\left(x_{1}\right)\right)^{k}\left(d_{G_{2}}\left(x_{1}\right) d_{G_{2}}\left(x_{2}\right)\right)^{l}\right] \\
& \leq 2^{2 m+k} \sum_{\substack{m, k, l \\
m, k, l m+k+l=\alpha}} P_{m, k, l}\left[\Delta\left(G_{1}\right)^{2 m} \Delta\left(G_{2}\right)^{2 l} M_{1}^{k}\left(G_{1}\right) M_{1}^{k}\left(G_{2}\right)\right] \\
& \sum B_{5}=\sum_{s_{1} s_{2} \notin E_{G_{1}}} \sum_{x_{1} x_{2} \in E_{G_{2}}}\left[d\left(s_{1}, x_{1}\right) d\left(s_{2}, x_{2}\right)\right]^{\alpha} \\
& =\sum_{s_{1} s_{2} \notin E_{G_{1}}} \sum_{x_{1} x_{2} \in E_{G_{2}}}\left[\left(d_{R\left(G_{1}\right)}\left(s_{1}\right)+d_{G_{2}}\left(x_{1}\right)\right)\left(d_{R\left(G_{1}\right)}\left(s_{2}\right)+d_{G_{2}}\left(x_{2}\right)\right)\right]^{\alpha} \\
& =\sum_{s_{1} s_{2} \notin E_{G_{1}}} \sum_{x_{1} x_{2} \in E_{G_{2}}}\left[d_{R\left(G_{1}\right)}\left(s_{1}\right) d_{R\left(G_{1}\right)}\left(s_{2}\right)+d_{R\left(G_{1}\right)}\left(s_{1}\right) d_{G_{2}}\left(x_{2}\right)+d_{R\left(G_{1}\right)}\left(s_{2}\right) d_{G_{2}}\left(x_{1}\right)+d_{G_{2}}\left(x_{1}\right) d_{G_{2}}\left(x_{2}\right)\right]^{\alpha} \\
& =\sum_{s_{1} s_{2} \notin E_{G_{1}}} \sum_{x_{1} x_{2} \in E_{G_{2}}}\left[\sum_{\substack{m, k, l \\
m, k, l m+k+l=\alpha}} P_{m, k, l}\left(4 d_{G_{1}}\left(s_{1}\right) d_{G_{1}}\left(s_{2}\right)\right)^{m}\left(2 d_{G_{1}}\left(s_{1}\right) d_{G_{2}}\left(x_{2}\right)+d_{G_{1}}\left(s_{2}\right) d_{G_{2}}\left(x_{1}\right)\right)^{k}\left(d_{G_{2}}\left(x_{1}\right) d_{G_{2}}\left(x_{2}\right)\right)^{l}\right] \\
& \leq 2^{2 m+k} \sum_{\substack{m, k, l \\
m, k, l m+k+l=\alpha}} P_{m, k, l}\left[\Delta\left(G_{1}\right)^{2 m} \Delta\left(G_{2}\right)^{2 l} \bar{M}_{1}^{k}\left(G_{1}\right) M_{1}^{k}\left(G_{2}\right)\right], \\
& \sum B_{6}=\sum_{s_{1} s_{2} \in E_{G_{1}}} \sum_{x_{1} x_{2} \notin E_{G_{2}}}\left[d\left(s_{1}, x_{1}\right) d\left(s_{2}, x_{2}\right)\right]^{\alpha} \\
& =\sum_{s_{1} s_{2} \notin E_{G_{1}}} \sum_{x_{1} x_{2} \in E_{G_{2}}}\left[\left(d_{R\left(G_{1}\right)}\left(s_{1}\right)+d_{G_{2}}\left(x_{1}\right)\right)\left(d_{R\left(G_{1}\right)}\left(s_{2}\right)+d_{G_{2}}\left(x_{2}\right)\right)\right]^{\alpha}
\end{aligned}
$$




$$
\begin{aligned}
& =\sum_{s_{1} s_{2} \notin E_{G_{1}}} \sum_{x_{1} x_{2} \notin E_{G_{2}}}\left[d_{R\left(G_{1}\right)}\left(s_{1}\right) d_{R\left(G_{1}\right)}\left(s_{2}\right)+d_{R\left(G_{1}\right)}\left(s_{1}\right) d_{G_{2}}\left(x_{2}\right)+d_{R\left(G_{1}\right)}\left(s_{2}\right) d_{G_{2}}\left(x_{1}\right)+d_{G_{2}}\left(x_{1}\right) d_{G_{2}}\left(x_{2}\right)\right]^{\alpha} \\
& =\sum_{s_{1} s_{2} \notin E_{G_{1}}} \sum_{x_{1} x_{2} \notin E_{G_{2}}}\left[\sum_{\substack{m, k, l \\
m, k, l m+k+l=\alpha}} P_{m, k, l}\left(4 d_{G_{1}}\left(s_{1}\right) d_{G_{1}}\left(s_{2}\right)\right)^{m}\left(2 d_{G_{1}}\left(s_{1}\right) d_{G_{2}}\left(x_{2}\right)+d_{G_{1}}\left(s_{2}\right) d_{G_{2}}\left(x_{1}\right)\right)^{k}\left(d_{G_{2}}\left(x_{1}\right) d_{G_{2}}\left(x_{2}\right)\right)^{l}\right] \\
& \leq 2^{2 m+k} \sum_{\substack{m, k, l \\
m, k, l m+k+l=\alpha}} P_{m, k, l}\left[\Delta\left(G_{1}\right)^{2 m} \Delta\left(G_{2}\right)^{2 l} M_{1}^{k}\left(G_{1}\right) \bar{M}_{1}^{k}\left(G_{2}\right)\right] \\
& \sum B_{7}=\sum_{s_{1} s_{2} \in E_{G_{1}}} \sum_{x_{1} x_{2} \in E_{G_{2}}}\left[d\left(s_{1}, x_{1}\right) d\left(s_{2}, x_{2}\right)\right]^{\alpha} \\
& =\sum_{s_{1} s_{2} \notin E_{G_{1}}} \sum_{x_{1} x_{2} \in E_{G_{2}}}\left[\left(d_{R\left(G_{1}\right)}\left(s_{1}\right)+d_{G_{2}}\left(x_{1}\right)\right)\left(d_{R\left(G_{1}\right)}\left(s_{2}\right)+d_{G_{2}}\left(x_{2}\right)\right)\right]^{\alpha} \\
& =\sum_{s_{1} s_{2} \notin E_{G_{1}}} \sum_{x_{1} x_{2} \notin E_{G_{2}}}\left[d_{R\left(G_{1}\right)}\left(s_{1}\right) d_{R\left(G_{1}\right)}\left(s_{2}\right)+d_{R\left(G_{1}\right)}\left(s_{1}\right) d_{G_{2}}\left(x_{2}\right)+d_{R\left(G_{1}\right)}\left(s_{2}\right) d_{G_{2}}\left(x_{1}\right)+d_{G_{2}}\left(x_{1}\right) d_{G_{2}}\left(x_{2}\right)\right]^{\alpha} \\
& =\sum_{s_{1} s_{2} \notin E_{G_{1}}} \sum_{x_{1} x_{2} \notin E_{G_{2}}}\left[\sum_{\substack{m, k, l \\
m, k, l m+k+l=\alpha}} P_{m, k, l}\left(4 d_{G_{1}}\left(s_{1}\right) d_{G_{1}}\left(s_{2}\right)\right)^{m}\left(2 d_{G_{1}}\left(s_{1}\right) d_{G_{2}}\left(x_{2}\right)+d_{G_{1}}\left(s_{2}\right) d_{G_{2}}\left(x_{1}\right)\right)^{k}\left(d_{G_{2}}\left(x_{1}\right) d_{G_{2}}\left(x_{2}\right)\right)^{l}\right] \\
& \leq 2^{2 m+k} \sum_{\substack{m, k, l \\
m, k, l m+k+l=\alpha}} P_{m, k, l}\left[\Delta\left(G_{1}\right)^{2 m} \Delta\left(G_{2}\right)^{2 l} \bar{M}_{1}^{k}\left(G_{1}\right) \bar{M}_{1}^{k}\left(G_{2}\right)\right], \\
& \sum B=\sum_{\substack{m, k, l \\
m, k, l m+k+l=\alpha}} P_{m, k, l} 2^{2 m+k}\left[M_{1}^{2 m+k}\left(G_{1}\right) \bar{\chi}_{k}\left(G_{2}\right) \Delta\left(G_{2}\right)^{2 l}\right]+\Delta\left(G_{1}\right)^{2 m}\left[M_{1}^{2 l+k}\left(G_{2}\right)\left(\chi_{k}\left(G_{1}\right)+\bar{\chi}_{k}\left(G_{1}\right)\right)\right] \\
& +\Delta\left(G_{2}\right)^{2 l}\left(M_{1}^{k}\left(G_{1}\right)+\bar{M}_{1}^{k}\left(G_{1}\right)\right)\left(M_{1}^{k}\left(G_{2}\right)+\bar{M}_{1}^{k}\left(G_{2}\right)\right), \\
& \sum C=\sum_{i=1}^{3} C_{i} \\
& \sum C_{1}=\sum_{\substack{s_{1} s_{2} \in E\left(R\left(G_{1}\right)\right) \\
\text { s } \in V\left(G_{1}\right) \\
s_{2} \in V\left(R\left(G_{1}\right)-V\left(G_{1}\right)\right)}}\left(\sum_{x_{1}, x_{2} \in V_{G_{2}}}+\sum_{x_{1}, x_{2} \notin V_{G_{2}}}\right)\left[d\left(s_{1}, x\right) d\left(s_{2}, x\right)\right]^{\text {alpha }} \\
& =\sum_{\substack{s_{1} s_{2} \in E\left(R\left(G_{1}\right)\right) \\
\text { s } \\
s_{1} \in V\left(G_{1}\right)}}\left(\sum_{x_{1}, x_{2} \in V_{G_{2}}}+\sum_{\left.\left.x_{1}, x_{2} \notin V_{G_{1}}\right)-V\left(G_{1}\right)\right)}\right)\left[\left(d_{R}\left(s_{1}\right)+d_{G_{2}}\left(x_{1}\right)\right)\left(d_{R}\left(s_{2}\right)\right)\right]^{\alpha} \\
& =\sum_{\substack{s_{1} s_{2} \in E\left(R\left(G_{1}\right)\right) \\
s_{1} \in V\left(G_{1}\right) \\
s_{2} \notin V\left(R\left(G_{1}\right)-V\left(G_{1}\right)\right)}}\left(\sum_{x_{1}, x_{2} \in V_{G_{2}}}+\sum_{x_{1}, x_{2} \notin V_{G_{2}}}\right)\left[\left(d_{R}\left(s_{1}\right)+d_{G_{2}}\left(x_{1}\right)\right)\left(d_{R}\left(s_{2}\right)\right)\right]^{\alpha}
\end{aligned}
$$




$$
\begin{aligned}
& =\sum_{\substack{s_{1} s_{2} \in E\left(R\left(G_{1}\right)\right) \\
s_{1} \in V\left(G_{1}\right) \\
s_{2} \in V\left(R\left(G_{1}\right)-V\left(G_{1}\right)\right)}}\left(\sum_{x_{1}, x_{2} \in V_{G_{2}}}+\sum_{x_{1}, x_{2} \notin V_{G_{2}}}\right)\left[\left(d_{R\left(G_{1}\right)}\left(s_{1}\right)\left(d_{R}\left(s_{2}\right)\right)+d_{G_{2}}\left(x_{1}\right)\right)\left(d_{R\left(G_{1}\right)}\left(s_{2}\right)\right)\right]^{\alpha} \\
& =\sum_{\substack{s_{1} s_{2} \in E\left(R\left(G_{1}\right)\right) \\
s_{1} \in V\left(G_{1}\right) \\
s_{2} \in V\left(R\left(G_{1}\right)-V\left(G_{1}\right)\right)}}\left(\sum_{x_{1}, x_{2} \in V_{G_{2}}}+\sum_{x_{1}, x_{2} \notin V_{G_{2}}}\right)\left[\sum_{i=0}^{\alpha}\left(\begin{array}{c}
\alpha \\
i
\end{array}\right)\left(d_{R\left(G_{1}\right)}\left(s_{1}\right)\left(d_{R}\left(s_{2}\right)\right) d_{G_{2}}\left(x_{1}\right)\right)^{i}\left(d_{R\left(G_{1}\right)}\left(s_{2}\right)\right)^{i}\right] \\
& s_{2} \in V\left(R\left(G_{1}\right)-V\left(G_{1}\right)\right) \\
& \leq \sum_{i=0}^{\alpha}\left(\begin{array}{c}
\alpha \\
i
\end{array}\right)\left[M_{2}^{n-i}\left(R\left(G_{1}\right)\right) \Delta^{i}\left(R\left(G_{1}\right)\right)\left(M_{1}^{i}\left(G_{2}\right)+\bar{M}_{1}^{i}\left(G_{2}\right)\right)\right], \\
& \sum C_{2}=\sum_{\substack{s_{1} s_{2} \notin E\left(R\left(G_{1}\right)\right) \\
s_{1} \in V\left(G_{1}\right) \\
s_{2} \in V\left(R\left(G_{1}\right)-V\left(G_{1}\right)\right)}} \sum_{x \in V_{G_{2}}}\left[d\left(s_{1}, x\right) d\left(s_{2}, x\right)\right]^{\alpha}=\sum_{\substack{s_{1} s_{2} \notin E\left(R\left(G_{1}\right)\right) \\
s_{1} \in V\left(G_{1}\right) \\
s_{2} \in V\left(R\left(G_{1}\right)-V\left(G_{1}\right)\right)}} \sum_{x \in V_{G_{2}}}\left[\left(d_{R}\left(s_{1}\right)+d_{G_{2}}(x)\right)\left(d_{R}\left(s_{2}\right)\right)\right]^{\alpha} \\
& =\sum_{\substack{s_{1} s_{2} \notin E\left(R\left(G_{1}\right)\right) \\
\text { s } \in V\left(G_{1}\right) \\
s_{2} \in V\left(R\left(G_{1}\right)-V\left(G_{1}\right)\right)}} \sum_{x \in V_{G_{2}}}\left[\left(d_{R\left(G_{1}\right)}\left(s_{1}\right)\left(d_{R}\left(s_{2}\right)\right)+d_{G_{2}}(x)\right)\left(d_{R\left(G_{1}\right)}\left(s_{2}\right)\right)\right]^{\alpha} \\
& =\sum_{\substack{s_{1} s_{2} \notin E\left(R\left(G_{1}\right)\right) \\
s_{1} \in V\left(G_{1}\right)}} \sum_{x \in V_{G_{2}}}\left[\sum_{i=0}^{\alpha}\left(\begin{array}{c}
\alpha \\
i
\end{array}\right)\left(d_{R\left(G_{1}\right)}\left(s_{1}\right)\left(d_{R}\left(s_{2}\right)\right)^{n-i} d_{G_{2}}(x)\right)^{i}\left(d_{R\left(G_{1}\right)}\left(s_{2}\right)\right)^{i}\right] \\
& \begin{array}{c}
s_{1} \in\left(G_{1}\right) \\
s_{2} \in V\left(R\left(G_{1}\right)-V\left(G_{1}\right)\right)
\end{array} \\
& \leq \sum_{i=0}^{\alpha}\left(\begin{array}{c}
\alpha \\
i
\end{array}\right)\left[\alpha_{1}^{n-i} M_{1}^{i}\left(G_{2}\right) \Delta^{i}\left(R\left(G_{1}\right)\right)\right] \\
& \sum C_{3}=\sum_{\substack{s_{1} s_{2} \notin E\left(R\left(G_{1}\right)\right) \\
\text { sis }\left(R\left(G_{1}\right) \\
s_{2} \in V\left(R\left(G_{1}\right)-V\left(G_{1}\right)\right)\right.}}\left(\sum_{x_{1}, x_{2} \in V_{G_{2}}}+\sum_{x_{1}, x_{2} \notin V_{G_{2}}}\right)\left[d\left(s_{1}, x\right) d\left(s_{2}, x\right)\right]^{\alpha} \\
& =\sum_{\substack{s_{1} s_{2} \notin E\left(R\left(G_{1}\right)\right) \\
s_{1} \in V\left(G_{1}\right) \\
s_{2} \in V\left(R\left(G_{1}\right)-V\left(G_{1}\right)\right)}}\left(\sum_{x_{1}, x_{2} \in V_{G_{2}}}+\sum_{x_{1}, x_{2} \notin V_{G_{2}}}\right)\left[\left(d_{R}\left(s_{1}\right)+d_{G_{2}}\left(x_{1}\right)\right)\left(d_{R}\left(s_{2}\right)\right)\right]^{\alpha} \\
& =\sum_{\substack{s_{1} s_{2} \notin E\left(R\left(G_{1}\right)\right) \\
\text { s. } \\
s_{2} \in V\left(G_{1}\right)}}\left(\sum_{\left.\left.x_{1}, x_{2} \in V_{G_{2}}\right)-V\left(G_{1}\right)\right)}+\sum_{x_{1}, x_{2} \notin V_{G_{2}}}\right)\left[\left(d_{R}\left(s_{1}\right)+d_{G_{2}}\left(x_{1}\right)\right)\left(d_{R}\left(s_{2}\right)\right)\right]^{\alpha}
\end{aligned}
$$

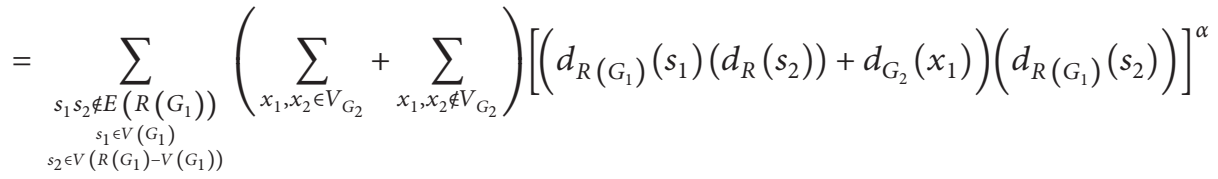




$$
\begin{aligned}
& =\sum_{\substack{s_{1} s_{2} \notin E\left(R\left(G_{1}\right)\right) \\
s_{1} \in V\left(G_{1}\right) \\
s_{2} \in V\left(R\left(G_{1}\right)-V\left(G_{1}\right)\right)}}\left(\sum_{x_{1}, x_{2} \in V_{G_{2}}}+\sum_{x_{1}, x_{2} \notin V_{G_{2}}}\right)\left[\sum_{i=0}^{\alpha}\left(\begin{array}{c}
\alpha \\
i
\end{array}\right)\left(d_{R\left(G_{1}\right)}\left(s_{1}\right)\left(d_{R}\left(s_{2}\right)\right)^{n-i} d_{G_{2}}\left(x_{1}\right)\right)^{i}\left(d_{R\left(G_{1}\right)}\left(s_{2}\right)\right)^{i}\right] \\
& \leq \sum_{i=0}^{\alpha}\left(\begin{array}{c}
\alpha \\
i
\end{array}\right)\left[\alpha_{1}^{n-i} \Delta^{i}\left(R\left(G_{1}\right)\right)\left(M_{1}^{i}\left(G_{2}\right)+\bar{M}_{1}^{i}\left(G_{2}\right)\right)\right] .
\end{aligned}
$$

We obtained upper bound by putting value of $\sum A, \sum B$, and $\sum C$ in equation (16). Similarly, lower bound can be $\mathrm{LB} \leq \bar{R}_{\alpha}\left(G_{1+Q} G_{2}\right) \leq \mathrm{UB}$, obtained using smallest degree of graphs $G_{1}$ and $G_{2}$.

Theorem 3. Let $G_{1+Q} G_{2}$ be a $Q$-sum graph, then its general Randic coindex $\bar{R}_{\alpha}\left(G_{1+Q} G_{2}\right)$ is given as

$$
\begin{aligned}
\mathrm{LB}= & \sum_{\substack{m, k, l \\
m, k, l m+k+l=\alpha}} P_{m, k, l}\left[M_{1}^{2 m+k}\left(G_{1}\right) \bar{\chi}_{k}\left(G_{2}\right) \delta\left(G_{2}\right)^{2 l}\right]+\delta\left(G_{1}\right)^{2 m} \\
& {\left[M_{1}^{2 l+k}\left(G_{2}\right)\left(\chi_{k}\left(G_{1}\right)+\bar{\chi}_{k}\left(G_{1}\right)\right)\right]+\delta\left(G_{2}\right)^{2 l}\left(M_{1}^{k}\left(G_{1}\right)+\bar{M}_{1}^{k}\left(G_{1}\right)\right)\left(M_{1}^{k}\left(G_{2}\right)+\bar{M}_{1}^{k}\left(G_{2}\right)\right) } \\
& +\sum_{i=0}^{\alpha}\left(\begin{array}{c}
\alpha \\
i
\end{array}\right) \delta^{i}\left(Q\left(G_{1}\right)\right)\left[M_{2}^{n-i}\left(Q\left(G_{1}\right)\right)\left(M_{1}^{i}\left(G_{2}\right)+\bar{M}_{1}^{i}\left(G_{2}\right)\right)\right]+\alpha_{1}^{n-i}\left(M_{1}^{i}\left(G_{2}\right)+\bar{M}_{1}^{i}\left(G_{2}\right)\right)+\alpha_{2} \\
\mathrm{UB}= & \sum_{m, k, l} P_{m, k, l}\left[M_{1}^{2 m+k}\left(G_{1}\right) \bar{\chi}_{k}\left(G_{2}\right) \Delta\left(G_{2}\right)^{2 l}\right] \\
& +\Delta\left(G_{1}\right)^{2 m}\left[M_{1}^{2 l+k}\left(G_{2}\right)\left(\chi_{k}\left(G_{1}\right)+\bar{\chi}_{k}\left(G_{1}\right)\right)\right]+\Delta\left(G_{2}\right)^{2 l}\left(M_{1}^{k}\left(G_{1}\right)+\bar{M}_{1}^{k}\left(G_{1}\right)\right) \\
& \left(M_{1}^{k}\left(G_{2}\right)+\bar{M}_{1}^{k}\left(G_{2}\right)\right)+\sum_{i=0}^{\alpha}\left(\begin{array}{c}
\alpha \\
i
\end{array}\right) \Delta^{i}\left(Q\left(G_{1}\right)\right) \\
& {\left[M_{2}^{n-i}\left(Q\left(G_{1}\right)\right)\left(M_{1}^{i}\left(G_{2}\right)+\bar{M}_{1}^{i}\left(G_{2}\right)\right)\right]+\alpha_{1}^{n-i}\left(M_{1}^{i}\left(G_{2}\right)+\bar{M}_{1}^{i}\left(G_{2}\right)\right)+\alpha_{2} . }
\end{aligned}
$$

Proof. Using equation (4), we have

Consider

$$
\bar{R}_{\alpha}\left(G_{1+Q} G_{2}\right)=\sum A+\sum B+\sum C .
$$

$$
\begin{aligned}
\sum A & =\sum_{s_{1}, s_{2} \in V} \sum_{\left(Q\left(G_{1}\right)-V\left(G_{1}\right)\right)} \sum_{x_{1}, x_{2} \in V_{G_{2}}}\left[d\left(s_{1}, x_{1}\right)\left(s_{2}, x_{2}\right)\right]^{\alpha} \\
& =\sum_{s_{1}, s_{2} \in V} \sum_{\left(Q\left(G_{1}\right)-V\left(G_{1}\right)\right)} \sum_{x_{1}, x_{2} \in V_{G_{2}}}\left[d_{Q\left(G_{1}\right)}\left(s_{1}\right) d_{Q\left(G_{1}\right)}\left(s_{2}\right)\right]=\alpha_{2} .
\end{aligned}
$$

The value of $\sum B$ follows from equation (17), 


$$
\begin{aligned}
& \sum C=\sum_{i=1}^{3} C_{i} \\
& \sum C_{1}=\sum_{\substack{s_{1} s_{2} \in E\left(Q\left(G_{1}\right)\right) \\
\text { s. } \\
s_{2} \in V\left(G_{1}\right)}}\left(\sum_{\left.\left.x_{1}, x_{2} \in V_{G_{1}}\right)-V\left(G_{1}\right)\right)}+\sum_{x_{1}, x_{2} \notin V_{G_{2}}}\right)\left[d\left(s_{1}, x\right) d\left(s_{2}, x\right)\right]^{\alpha} \\
& =\sum_{\substack{s_{1} s_{2} \notin E\left(Q\left(G_{1}\right)\right) \\
\text { s.t. } \\
s_{2} \in V\left(Q\left(G_{1}\right)-V\left(G_{1}\right)\right)}}\left(\sum_{x_{1}, x_{2} \in V_{G_{2}}}+\sum_{x_{1}, x_{2} \notin V_{G_{2}}}\right)\left[\left(d_{Q}\left(s_{1}\right)+d_{G_{2}}\left(x_{1}\right)\right)\left(d_{Q}\left(s_{2}\right)\right)\right]^{\alpha} \\
& =\sum_{\substack{s_{1} s_{2} \notin E\left(Q\left(G_{1}\right)\right) \\
s_{1} \in V\left(G_{1}\right) \\
s_{2} \in V\left(Q\left(G_{1}\right)-V\left(G_{1}\right)\right)}}\left(\sum_{x_{1}, x_{2} \in V_{G_{2}}}+\sum_{x_{1}, x_{2} \notin V_{G_{2}}}\right)\left[\left(d_{Q}\left(s_{1}\right)+d_{G_{2}}\left(x_{1}\right)\right)\left(d_{Q}\left(s_{2}\right)\right)\right] \\
& =\sum_{\substack{\left.s_{1} s_{2} \notin E\left(Q\left(G_{1}\right)\right) \\
\text { siteV(G }\right) \\
s_{2} \in V\left(Q\left(G_{1}\right)-V\left(G_{1}\right)\right)}}\left(\sum_{x_{1}, x_{2} \in V_{G_{2}}}+\sum_{x_{1}, x_{2} \notin V_{G_{2}}}\right)\left[\left(d_{Q\left(G_{1}\right)}\left(s_{1}\right)\left(d_{Q}\left(s_{2}\right)\right)+d_{G_{2}}\left(x_{1}\right)\right)\left(d_{Q\left(G_{1}\right)}\left(s_{2}\right)\right)\right]^{\alpha} \\
& =\sum_{\substack{s_{1} s_{2} \notin E\left(Q\left(G_{1}\right)\right) \\
\text { s.t. } \\
s_{2} \in V\left(Q\left(G_{1}\right)-V\left(G_{1}\right)\right)}}\left(\sum_{x_{1}, x_{2} \in V_{G_{2}}}+\sum_{x_{1}, x_{2} \notin V_{G_{2}}}\right)\left[\sum_{i=0}^{\alpha}\left(\begin{array}{c}
\alpha \\
i
\end{array}\right)\left(d_{Q\left(G_{1}\right)}\left(s_{1}\right)\left(d_{Q}\left(s_{2}\right)\right)^{n-i} d_{G_{2}}\left(x_{1}\right)\right)^{i}\left(d_{Q\left(G_{1}\right)}\left(s_{2}\right)\right)^{i}\right] \\
& \leq \sum_{i=0}^{\alpha}\left(\begin{array}{c}
\alpha \\
i
\end{array}\right)\left[M_{2}^{n-i}\left(Q\left(G_{1}\right)\right) \Delta^{i}\left(Q\left(G_{1}\right)\right)\left(M_{1}^{i}\left(G_{2}\right)+\bar{M}_{1}^{i}\left(G_{2}\right)\right)\right], \\
& \sum C_{2}=\sum_{\substack{s_{1} s_{2} \notin E\left(Q\left(G_{1}\right)\right) \\
s_{1} \in V\left(G_{1}\right) \\
s_{2} \in V\left(Q\left(G_{1}\right)-V\left(G_{1}\right)\right)}} \sum_{\substack{x \in V_{G_{2}} \\
s_{1}}}\left[d\left(s_{1}, x\right) d\left(s_{2}, x\right)\right]^{\alpha}=\sum_{\substack{s_{1} s_{2} \notin E\left(Q\left(G_{1}\right)\right) \\
s_{1} \in V\left(G_{1}\right) \\
s_{2} \in V\left(Q\left(G_{1}\right)-V\left(G_{1}\right)\right)}} \sum_{x \in V_{G_{2}}}\left[\left(d_{Q}\left(s_{1}\right)+d_{G_{2}}(x)\right)\left(d_{Q}\left(s_{2}\right)\right)\right]^{\alpha} \\
& =\sum_{\substack{s_{1} s_{2} \notin E\left(Q\left(G_{1}\right)\right) \\
\text { s. } \in V\left(G_{1}\right) \\
s_{2} \in V\left(Q\left(G_{1}\right)-V\left(G_{1}\right)\right)}} \sum_{x \in V_{G_{2}}}\left[\left(d_{Q\left(G_{1}\right)}\left(s_{1}\right)\left(d_{Q}\left(s_{2}\right)\right)+d_{G_{2}}(x)\right)\left(d_{Q\left(G_{1}\right)}\left(s_{2}\right)\right)\right]^{\alpha} \\
& =\sum_{\substack{s_{1} s_{2} \notin E\left(Q\left(G_{1}\right)\right) \\
s_{1} \in V\left(G_{1}\right) \\
s_{2} \in V\left(Q\left(G_{1}\right)-V\left(G_{1}\right)\right)}} \sum_{x \in V_{G_{2}}}\left[\sum_{i=0}^{\alpha}\left(\begin{array}{c}
\alpha \\
i
\end{array}\right)\left(d_{Q\left(G_{1}\right)}\left(s_{1}\right)\left(d_{Q}\left(s_{2}\right)\right)^{n-i} d_{G_{2}}(x)\right)^{i}\left(d_{Q\left(G_{1}\right)}\left(s_{2}\right)\right)^{i}\right] \\
& \leq \sum_{i=0}^{\alpha}\left(\begin{array}{c}
\alpha \\
i
\end{array}\right)\left[\alpha_{1}^{n-i} M_{1}^{i}\left(G_{2}\right) \Delta^{i}\left(Q\left(G_{1}\right)\right)\right] \\
& \sum C_{3}=\sum_{\substack{s_{1} s_{2} \notin E\left(Q\left(G_{1}\right)\right) \\
\text { s.te } \\
s_{2} \in V\left(Q\left(G_{1}\right)\right.}}\left(\sum_{\left.\left.x_{1}, x_{2} \in V_{G_{2}}\right)-V\left(G_{1}\right)\right)}+\sum_{x_{1}, x_{2} \notin V_{G_{2}}}\right)\left[d\left(s_{1}, x\right) d\left(s_{2}, x\right)\right]^{\alpha}
\end{aligned}
$$


TABLE 1: Bounded values of certain F-sum graphs.

\begin{tabular}{lccc}
\hline$F$-sum operation & Lower bounds & Exact values & Upper bounds \\
$\bar{R}_{2}\left(P_{3+S} P_{2}\right)$ & 324 & 784 & 1076 \\
$\bar{R}_{2}\left(P_{3+R} P_{2}\right)$ & 1640 & 2502 & 6784 \\
$\bar{R}_{2}\left(P_{3+Q} P_{2}\right)$ & 958 & 1432 & 1624 \\
$\bar{R}_{2}\left(P_{3+T} P_{2}\right)$ & 3604 & 3770 & 10692 \\
\hline
\end{tabular}

$$
\begin{aligned}
& =\sum_{\substack{s_{1} s_{2} \notin E\left(Q\left(G_{1}\right)\right) \\
s_{1} \in V\left(G_{1}\right) \\
s_{2} \in V\left(Q\left(G_{1}\right)-V\left(G_{1}\right)\right)}}\left(\sum_{x_{1}, x_{2} \in V_{G_{2}}}+\sum_{x_{1}, x_{2} \notin V_{G_{2}}}\right)\left[\left(d_{Q}\left(s_{1}\right)+d_{G_{2}}\left(x_{1}\right)\right)\left(d_{Q}\left(s_{2}\right)\right)\right]^{\alpha} \\
& =\sum_{\substack{s_{1} s_{2} \notin E\left(Q\left(G_{1}\right)\right) \\
\text { s. } \\
s_{2} \in V\left(G_{1}\right)}}\left(\sum_{x_{1}, x_{2} \in V_{G_{2}}}+\sum_{\left.\left.x_{1}, x_{2} \notin V_{G_{2}}\right)-V\left(G_{1}\right)\right)}\right)\left[\left(d_{Q}\left(s_{1}\right)+d_{G_{2}}\left(x_{1}\right)\right)\left(d_{Q}\left(s_{2}\right)\right)\right]^{\alpha} \\
& =\sum_{\substack{s_{1} s_{2} \notin E\left(Q\left(G_{1}\right)\right) \\
\text { s. } \\
s_{2} \in V\left(G_{1}\right)}}\left(\sum_{\left.x_{1}, x_{2} \in V_{G_{2}}\right)}+\sum_{x_{1}, x_{2} \notin V_{G_{2}}}\right)\left[\left(d_{\left.Q\left(G_{1}\right)\right)}\left(s_{1}\right)\left(d_{Q}\left(s_{2}\right)\right)+d_{G_{2}}\left(x_{1}\right)\right)\left(d_{Q\left(G_{1}\right)}\left(s_{2}\right)\right)\right]^{\alpha} \\
& =\sum_{\left.\substack{s_{1} s_{2} \notin E\left(Q\left(G_{1}\right)\right) \\
\text { s. } \\
s_{2} \in V\left(G_{1}\right)}\left(G_{1}\right)-V\left(G_{1}\right)\right)}\left(\sum_{x_{1}, x_{2} \in V_{G_{2}}}+\sum_{x_{1}, x_{2} \notin V_{G_{2}}}\right)\left[\sum_{i=0}^{\alpha}\left(\begin{array}{c}
\alpha \\
i
\end{array}\right)\left(d_{Q\left(G_{1}\right)}\left(s_{1}\right)\left(d_{Q}\left(s_{2}\right)\right)^{n-i} d_{G_{2}}\left(x_{1}\right)\right)^{i}\left(d_{Q\left(G_{1}\right)}\left(s_{2}\right)\right)^{i}\right] \\
& \leq \sum_{i=0}^{\alpha}\left(\begin{array}{c}
\alpha \\
i
\end{array}\right)\left[\alpha_{1}^{n-i} \Delta^{i}\left(Q\left(G_{1}\right)\right)\left(M_{1}^{i}\left(G_{2}\right)+\bar{M}_{1}^{i}\left(G_{2}\right)\right)\right] .
\end{aligned}
$$

We obtained upper bound by putting value of $\sum A, \sum B$, and $\sum C$ in equation (20). Similarly, lower bound can be obtained using smallest degree of graphs $G_{1}$ and $G_{2}$.

$$
\mathrm{LB} \leq \bar{R}_{\alpha}\left(G_{1+T} G_{2}\right) \leq \mathrm{UB},
$$

Theorem 4. Let $G_{1+T} G_{2}$ be a T-sum graph, then its general Randic coindex $\bar{R}_{\alpha}\left(G_{1+T} G_{2}\right)$ is given as

$$
\begin{aligned}
\mathrm{LB}= & \sum_{\substack{m, k, l \\
m, k, l m+k+l=\alpha}} P_{m, k, l} 2^{2 m+k}\left[M_{1}^{2 m+k}\left(G_{1}\right) \bar{\chi}_{k}\left(G_{2}\right) \delta\left(G_{2}\right)^{2 l}\right]+\delta\left(G_{1}\right)^{2 m} \\
& {\left[M_{1}^{2 l+k}\left(G_{2}\right)\left(\chi_{k}\left(G_{1}\right)+\bar{\chi}_{k}\left(G_{1}\right)\right)\right]+\delta\left(G_{2}\right)^{2 l}\left(M_{1}^{k}\left(G_{1}\right)+\bar{M}_{1}^{k}\left(G_{1}\right)\right)\left(M_{1}^{k}\left(G_{2}\right)+\bar{M}_{1}^{k}\left(G_{2}\right)\right) } \\
& +\sum_{i=0}^{\alpha}\left(\begin{array}{c}
\alpha \\
i
\end{array}\right) \delta^{i}\left(T\left(G_{1}\right)\right)\left[M_{2}^{n-i}\left(T\left(G_{1}\right)\right)\left(M_{1}^{i}\left(G_{2}\right)+\bar{M}_{1}^{i}\left(G_{2}\right)\right)\right]+\alpha_{1}^{n-i}\left(M_{1}^{i}\left(G_{2}\right)+\bar{M}_{1}^{i}\left(G_{2}\right)\right) \alpha_{2}, \\
\mathrm{UB}= & \sum_{\substack{m, k, l \\
m, k, l m+k+l=\alpha}} P_{m, k, l} 2^{2 m+k}\left[M_{1}^{2 m+k}\left(G_{1}\right) \bar{\chi}_{k}\left(G_{2}\right) \Delta\left(G_{2}\right)^{2 l}\right]+\Delta\left(G_{1}\right)^{2 m} \\
& {\left[M_{1}^{2 l+k}\left(G_{2}\right)\left(\chi_{k}\left(G_{1}\right)+\bar{\chi}_{k}\left(G_{1}\right)\right)\right]+\Delta\left(G_{2}\right)^{2 l}\left(M_{1}^{k}\left(G_{1}\right)+\bar{M}_{1}^{k}\left(G_{1}\right)\right)\left(M_{1}^{k}\left(G_{2}\right)+\bar{M}_{1}^{k}\left(G_{2}\right)\right) } \\
& +\sum_{i=0}^{\alpha}\left(\begin{array}{c}
\alpha \\
i
\end{array}\right) \Delta^{i}\left(T\left(G_{1}\right)\right)\left[M_{2}^{n-i}\left(T\left(G_{1}\right)\right)\left(M_{1}^{i}\left(G_{2}\right)+\bar{M}_{1}^{i}\left(G_{2}\right)\right)\right]+\alpha_{1}^{n-i}\left(M_{1}^{i}\left(G_{2}\right)+\bar{M}_{1}^{i}\left(G_{2}\right)\right)+\alpha_{2} .
\end{aligned}
$$

It follows from Theorems 2 and 3. 


\section{Conclusion}

(i) In this paper, we calculated the upper and lower bounds of general Randic coindex for $F$-sum graphs such as $\bar{R}_{\alpha}\left(G_{1+S} G_{2}\right), \bar{R}_{\alpha}\left(G_{1+R} G_{2}\right), \bar{R}_{\alpha}\left(G_{1+Q} G_{2}\right)$, and $\bar{R}_{\alpha}\left(G_{1+T} G_{2}\right)$ in the form of Zagreb indices and coincides of their factor graphs.

(ii) We illustrate the results using two graphs $G_{1} \cong C_{n}$ and $G_{2} \cong P_{n}$, then bounded values of general Randic coindex are given in Table 1 .

\section{Data Availability}

In this paper, complete data are included. However, the reader may request the corresponding author for more details of the data.

\section{Conflicts of Interest}

The authors declare no conflicts of interest.

\section{References}

[1] A. R. Matamala and E. Estrada, "Generalised topological indices: optimisation methodology and physico-chemical interpretation," Chemical Physics Letters, vol. 410, no. 4-6, pp. 343-347, 2005.

[2] G. Rücker and C. Rucker, "On topological indices, boiling points, and cycloalkanes," Journal of Chemical Information and Computer Sciences, vol. 39, no. 5, pp. 788-802, 1999.

[3] K. Xu, M. Liu, K. C. Das, I. Gutman, and B. Furtula, “"A survey on graphs extremal with respect to distance-based topological indices," MATCH Commun," MATCH Communications in Mathematical and in Computer Chemistry, vol. 71, no. 3, pp. 461-508, 2014.

[4] H. Wiener, "Structural determination of paraffin boiling points," Journal of the American Chemical Society, vol. 69, no. 1, pp. 17-20, 1947.

[5] I. Gutman and N. Trinajstić, "Graph theory and molecular orbitals. Total $\varphi$-electron energy of alternant hydrocarbons," Chemical Physics Letters, vol. 17, no. 4, pp. 535-538, 1972.

[6] X. Li and J. Zheng, "A unified approach to the extremal trees for different indices," MATCH Communications in Mathematical and in Computer Chemistry, vol. 54, pp. 195-208, 2005.

[7] X. Li and Y. Shi, "A survey on the Randic index," MATCH Communications in Mathematical and in Computer Chemistry, vol. 59, no. 1, pp. 127-156, 2008.

[8] C. Delorme, O. Favaron, and D. Rautenbach, "On the Randić index,” Discrete Mathematics, vol. 257, no. 1, pp. 29-38, 2002.

[9] I. Gutman, B. Furtula, and V. Katanić, "Randić index and information," AKCE International Journal of Graphs and Combinatorics, vol. 15, no. 3, pp. 307-312, 2018.

[10] G. Arizmendi and O. Arizmendi, "Energy of a graph and Randic index," Linear Algebra and Its Applications, vol. 609, pp. 332-338, 2021.

[11] X. Li and Y. Yang, "Sharp bounds for the general Randic index," MATCH Communications in Mathematical and in Computer Chemistry, vol. 51, pp. 155-166, 2004.

[12] B. Furtula and I. Gutman, "Comparing energy and Randic energy," Macedonian Journal of Chemistry and Chemical Engineering, vol. 32, no. 1, pp. 117-123, 2013.
[13] J. Gao and M. Lu, "On the Randic index of unicyclic graphs," MATCH Communications in Mathematical and in Computer Chemistry, vol. 53, no. 2, pp. 377-384, 2005.

[14] X. Li, X. Wang, and B. Wei, "On the lower and upper bounds for general randic index of chemical $(n-m)$ graphs," MATCH Communications in Mathematical and in Computer Chemistry, vol. 52, pp. 157-166, 2004

[15] Y. Ma, S. Cao, Y. Shi, I. Gutman, M. Dehmer, and B. Furtula, "From the connectivity index to various Randic-type descriptors," MATCH Communications in Mathematical and in Computer Chemistry, vol. 80, pp. 85-106, 2018.

[16] I. Milovanovici, M. Matejic, and E. Milovanovi, "A note on the general zeroth-order Randic coindex of graphs," Contributions to Mathematics, vol. 1, pp. 17-21, 2020.

[17] I. Milovanovic, M. Matejic, E. Milovanovic, and A. Ali, "On some mathematical properties of the general zeroth order Randic coindex of graphs," Applied Mathematics, Informations and Mechanics, vol. 12, no. 2, pp. 75-82, 2020.

[18] W. Yan, B.-Y. Yang, and Y.-N. Yeh, "The behavior of Wiener indices and polynomials of graphs under five graph decorations," Applied Mathematics Letters, vol. 20, no. 3, pp. 290-295, 2007.

[19] M. Eliasi and B. Taeri, "Four new sums of graphs and their Wiener indices," Discrete Applied Mathematics, vol. 157, no. 4, pp. 794-803, 2009.

[20] D. Sarala, H. Deng, C. Natarajan, and S. K. Ayyaswamy, "F index of graphs based on four new operations related to the strong product," AKCE International Journal of Graphs and Combinatorics, pp. 1-13, 2018.

[21] M. Imran, S. Baby, H. M. A. Siddiqui, and M. K. Shafiq, "On the bounds of degree-based topological indices of the Cartesian product of F-sum of connected graphs," Journal of Inequalities and Applications, vol. 2017, no. 1, 14 pages, Article ID 305, 2017.

[22] X. Li, M. Ahmad, M. Javaid, M. Saeed, and J. B. Liu, "Bounds on general randic index for F-sum graphs," Journal of Mathematics, vol. 2020, Article ID 9129365, 17 pages, 2020.

[23] M. Javaid, M. Ibraheem, U. Ahmad, and Q. Zhu, "Computing bounds for second zagreb coindex of sum graphs," Mathematical Problems in Engineering, vol. 2021, Article ID 4671105, 19 pages, 2021.

[24] S. Akhter and M. Imran, "Computing the forgotten topological index of four operations on graphs," AKCE International Journal of Graphs and Combinatorics, vol. 14, no. 1, pp. 70-79, 2017.

[25] J. B. Liu, S. Javed, M. Javaid, and K. Shabbir, "Computing first general Zagreb index of operations on graphs," IEEE access, vol. 7, pp. 47494-47502, 2017.

[26] M. Javaid, U. Ali, and J. B. Liu, "Computing analysis for first Zagreb connection index and coindex of resultant graphs," Mathematical Problems in Engineering, vol. 2021, Article ID 6019517, 19 pages, 2021.

[27] B. Zhou and N. Trinajstić, "On general sum-connectivity index," Journal of Mathematical Chemistry, vol. 47, no. 1, pp. 210-218, 2010.

[28] B. Bollobas and P. Erdos, "Graphs of extremal weights," Ars Combinatoria, vol. 50, pp. 225-233, 1998. 